\title{
Review Article \\ Function, Structure, and Evolution of the Major Facilitator Superfamily: The LacY Manifesto
}

\author{
M. Gregor Madej \\ Department of Physiology, University of California, Los Angeles, CA 90095, USA \\ Correspondence should be addressed to M. Gregor Madej; gmadej@mednet.ucla.edu
}

Received 5 June 2014; Accepted 7 August 2014; Published 18 September 2014

Academic Editor: Luis Loura

Copyright (C) 2014 M. Gregor Madej. This is an open access article distributed under the Creative Commons Attribution License, which permits unrestricted use, distribution, and reproduction in any medium, provided the original work is properly cited.

\begin{abstract}
The major facilitator superfamily (MFS) is a diverse group of secondary transporters with members found in all kingdoms of life. A paradigm for MFS is the lactose permease (LacY) of Escherichia coli, which couples the stoichiometric translocation of a galactopyranoside and an $\mathrm{H}^{+}$across the cytoplasmic membrane. LacY has been the test bed for the development of many methods applied for the analysis of transport proteins. X-ray structures of an inward-facing conformation and the most recent structure of an almost occluded conformation confirm many conclusions from previous studies. Although structure models are critical, they are insufficient to explain the catalysis of transport. The clues to understanding transport are based on the principles of enzyme kinetics. Secondary transport is a dynamic process-static snapshots of X-ray crystallography describe it only partially. However, without structural information, the underlying chemistry is virtually impossible to conclude. A large body of biochemical/biophysical data derived from systematic studies of site-directed mutants in LacY suggests residues critically involved in the catalysis, and a working model for the symport mechanism that involves alternating access of the binding site is presented. The general concepts derived from the bacterial LacY are examined for their relevance to other MFS transporters.
\end{abstract}

\section{Introduction}

Proteins can act as molecular devices to convert one energy form to another through cycles of conformational transitions. Symporters, antiporters, transporters, carriers, or permeases are such molecular devices that catalyze substrate-specific equilibration and/or translocation of solutes across a biological membrane (Figure 1(a)). In 1955, when the existence of cell membranes themselves was still being questioned, let alone the existence of proteins that transport solutes specifically across them, Cohen and Rickenberg [1] found an inducible transport system for lactose in Escherichia coli, and it was subsequently found to be part of the famous lac operon [2]. This discovery was the first time that a transport function was associated with genetics and indicated that a protein might be involved.

In addition to regulatory loci, it is now widely known that the lac operon contains three structural genes: (i) the $Z$ gene encoding galactosidase, a cytosolic enzyme that catalyzes cleavage of lactose into galactose and glucose once it enters the cell; (ii) the $Y$ gene encoding LacY (aka, lactose or lac permease, lac carrier, and later the galactoside $/ \mathrm{H}^{+}$symporter); and (iii) the $A$ gene encoding galactoside transacetylase, a cytosolic enzyme that catalyzes acetylation of mainly thiogalactopyranosides with acetyl-CoA as the acetyl donor and has an unknown physiological function. With the advent of molecular biology, LacY became the first gene encoding a membrane protein to be cloned and sequenced [3] leading to overexpression of LacY [4]. This success in the early days of molecular biology opened the study of secondary active transport at the molecular level. Overexpression of $\mathrm{LacY}$ combined with the use of a highly specific photoaffinity label [5] led to the functional reconstitution of LacY into proteoliposomes [6-9]. It was then shown that purified LacY catalyzes all the translocation reactions typical of the transport system in vivo with comparable turnover numbers $[9,10]$.

Accordingly to the chemiosmotic concept of Peter Mitchell [11-13], an electrochemical $\mathrm{H}^{+}$gradient $\left(\Delta \widetilde{\mu}_{\mathrm{H}^{+}}\right)$ composed of an electrical (the membrane potential, $\Delta \psi$ ) and a chemical (a $\mathrm{pH}$ gradient, $\Delta \mathrm{pH}$ ) component drives the steady-state accumulation of a wide variety of substrates 
Active transport

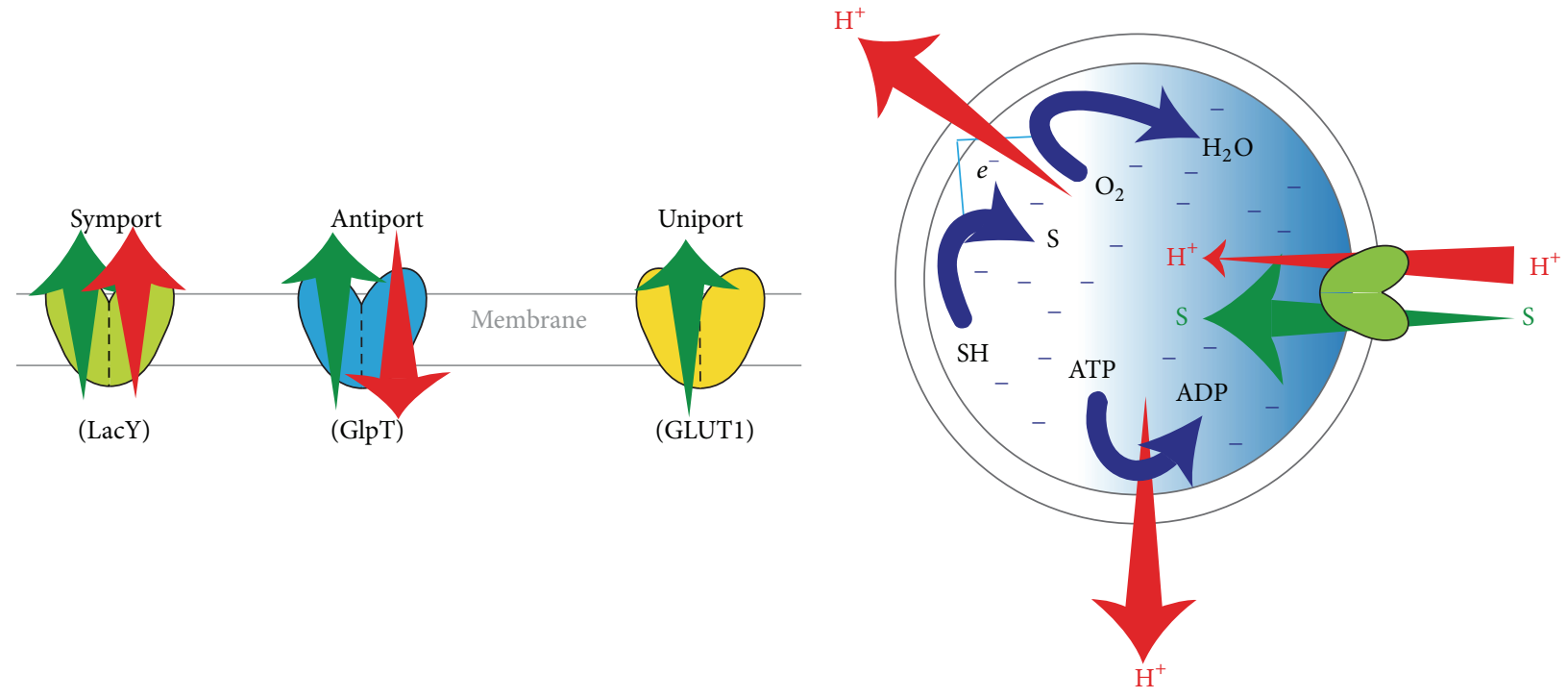

(a)

(b)

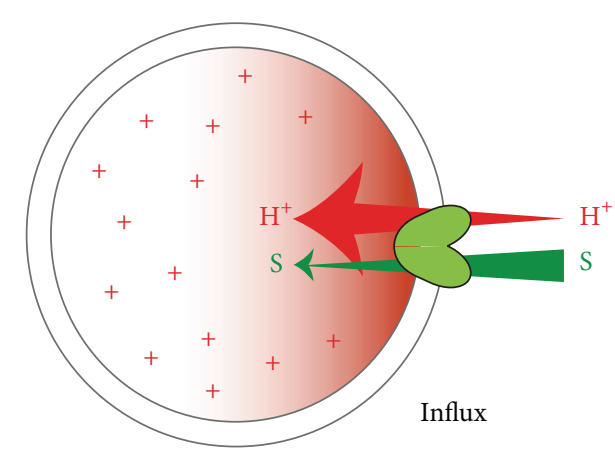

(c)

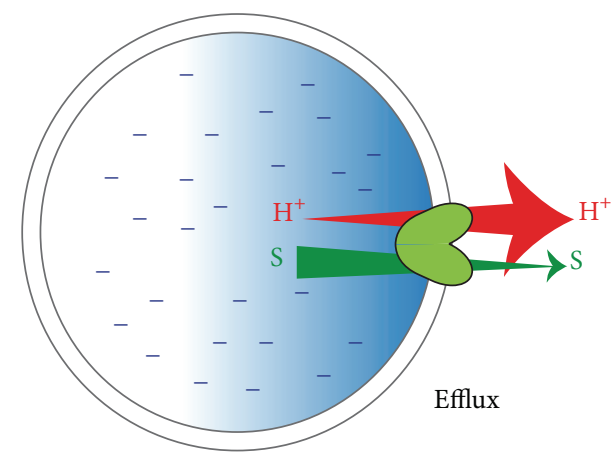

(d)

FIGURE 1: Transport reactions catalyzed by MFS. (a) Schematic illustration of active transports (symport, e.g., LacY, and antiport, e.g., GlpT) and facilitated diffusion (e.g., GLUT1). The red and green arrows indicate the transport directions of substrate and cosubstrate. (b) Uphill substrate (S) accumulation in response to $\Delta \widetilde{\mu}_{\mathrm{H}^{+}}$generated by either $\mathrm{H}^{+}$respiration or ATP hydrolysis by $\mathrm{F}_{1} \mathrm{~F}_{\mathrm{O}}-\mathrm{ATPase}$. (c) Uphill $\mathrm{H}^{+}$transport in response to an inwardly directed downhill lactose gradient (influx). (d) Uphill $\mathrm{H}^{+}$transport in response to an outwardly directed downhill lactose gradient (efflux). Substrate gradients generate $\Delta \widetilde{\mu}_{\mathrm{H}^{+}}$, the polarity of which depends upon the direction of the substrate concentration gradient.

according to the following relationship analogous to the Gibbs function:

$$
\Delta \tilde{\mu}_{\mathrm{H}^{+}}=-F \cdot \Delta \psi-2.3 R T \cdot \Delta \mathrm{pH}
$$

where $F$ is the Faraday constant, $R$ is the gas constant, and $T$ is the temperature.

A schematic representation of a chemiosmotic substrate transport is shown in Figure 1(b). An idealized cell or rightside-out (RSO) membrane vesicle (aka "Kabackosome") [14] pumping out $\mathrm{H}^{+}$by means of the membrane-embedded respiratory chain or hydrolysis of ATP by $F_{1} / F_{O}$ ATPase with the generation of a $\Delta \tilde{\mu}_{\mathrm{H}^{+}}$thereby creates an energetic sink for $\mathrm{H}^{+} . \mathrm{H}^{+}$-symporters such as $\mathrm{LacY}$ catalyze the obligatory coupled transport of a substrate and an $\mathrm{H}^{+}$, utilizing the free energy released from the thermodynamically favored movement (downhill) of $\mathrm{H}^{+}$to drive the accumulation of the substrate against its concentration gradient (uphill transport). It is particularly important to realize that coupling between sugar and $\mathrm{H}^{+}$translocation is obligatory. Thus, in the absence of $\Delta \widetilde{\mu}_{\mathrm{H}^{+}}$, downhill sugar influx or efflux will drive uphill $\mathrm{H}^{+}$transport with the generation of $\Delta \widetilde{\mu}_{\mathrm{H}^{+}}$, the polarity of which depends on the direction of the lactose gradient (Figures 1(c) and 1(d)).

Mitchell defined the measure of the potential energy stored as a combination of proton and voltage gradients as the proton-motive force $(\Delta p)$ in volts units $(\mathrm{V})$ to aid the comparison with oxidation-reduction (redox) midpoint potentials differences in the electron transfer chain complexes $\left(\Delta p[\mathrm{mV}]=-\left(\Delta \tilde{\mu}_{\mathrm{H}^{+}}\right) / F\right) ; \Delta \tilde{\mu}_{\mathrm{H}^{+}}=1 \mathrm{~kJ} \mathrm{~mol}^{-1}$ corresponds to $\Delta p=10.4 \mathrm{mV}$ (at $\left.25^{\circ} \mathrm{C}\right)$. Since $(-2.3 R T) / F \approx 59$, a $\Delta p$ of $-60 \mathrm{mV}$ in the form of either a $\Delta \psi$, a $\Delta \mathrm{pH}$, or a combination of both will theoretically generate a tenfold concentration 


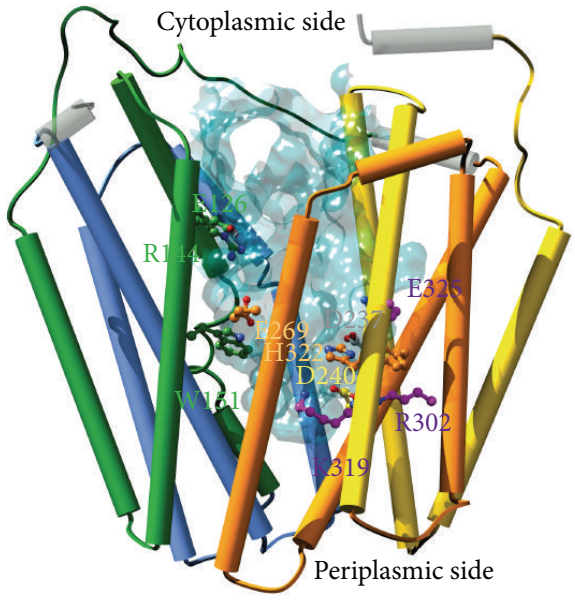

(a)

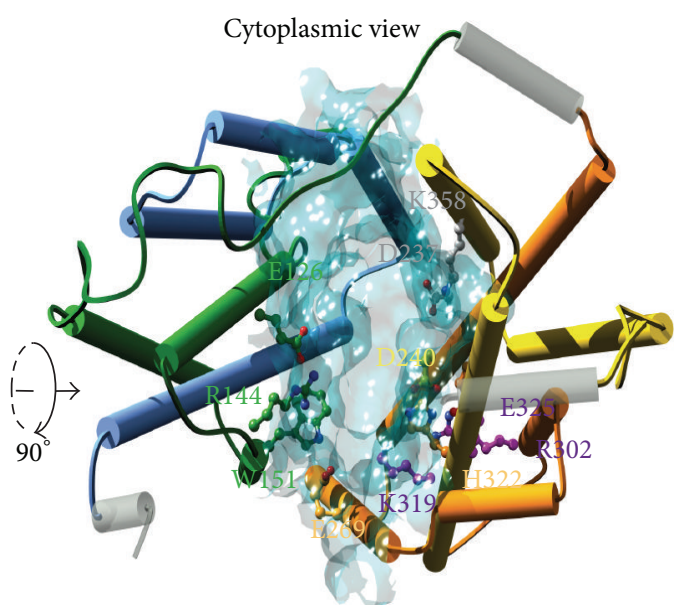

(b)

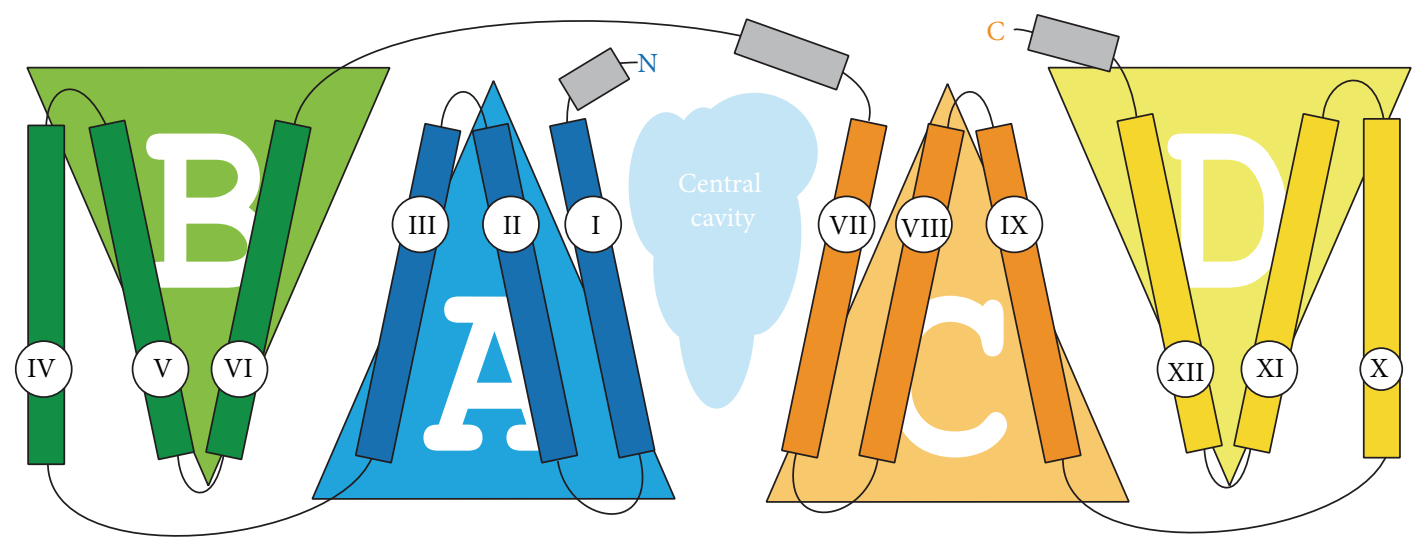

(c)

FIGURE 2: LacY structure. (a) Side view of the inward-facing conformation of LacY (PDB ID code: 2V8N). Helices are shown as rods and are colored according to the helix triplets (helices I-III: blue, IV-VI: green, VII-IX: orange, and X-XII: yellow). Critical residues are indicated (green and orange, residues involved in sugar binding; magenta, residues involved in $\mathrm{H}^{+}$translocation exclusively; gray, salt-bridged residues; and yellow, weakly salt-bridged residue; details are provided in the main text). The water-accessible surface of the cavity is shown as a light blue surface [calculated using the Computed Atlas of Surface Topography of proteins (CASTp) Web tool with a probe size of $1.4 \AA$ ]. (b) Cytoplasmic view; the color coding is the same as in A. (c) Topology of the internal structural repeats in the LacY; the helices are colored the same as in A and B blue. Inverted-topology repeats are indicated as triangles: blue: repeat unit A, green: repeat unit B, orange: repeat unit C, and yellow: repeat unit $\mathrm{D}$.

gradient of substrate, $-120 \mathrm{mV}$, a 100 -fold gradient, $-180 \mathrm{mV}$, a 1,000-fold gradient, and so forth.

\section{Major Facilitator Superfamily}

Lactose permease belongs to the major facilitator superfamily (MFS; Transporter Classification Database, TCDB \#2.A.1), the largest family of secondary transporters, present in all kingdoms of life. MFS transporters target a wide spectrum of substrates, including ions, carbohydrates, lipids, amino acids, and peptides, nucleosides, and other small molecules [40-42]. One remarkable characteristic of the MFS is the high sequence variety within the superfamily. The sequence identity ranges around $12-18 \%$ but regions of functional similarity (e.g., substrate- or $\mathrm{H}^{+}$-binding sites) align for only very closely related MFS transporters [43, 44]. A hydrophobic amino acid content of $60-70 \%$ of most MFS members, high $\alpha$-helix content, and the inherent symmetry of the proteins with regard to helix kinks and bends provides nonspecific overlapping of residues and probably accounts for the reported similarities [45]. Nevertheless, the MFS is subgrouped into 74 subfamilies based on phylogenetic analysis, postulated substrate specificity, and expected transport mode [42].

2.1. Architecture of the MFS Transporters. Many secondary active transporters reveal unique structural folds referred to as the LeuT [46], NhaA [47], or MFS [15, 20] types. Despite the very distinctive structural organization in each of the three types, two characteristics are conserved: discontinuous transmembrane helices and pseudosymmetries between structural motifs [48]. X-ray structure coordinates available for MFS transporters (Table 1 [15-36]) with low sequence similarity and distinct substrate specificities exhibit 
TABLE 1: Structural information for MFS transporters. Resolution limits and $R_{\text {free }}$ are stated only for the data sets with the highest resolution (PDB code in bold).

\begin{tabular}{|c|c|c|c|c|c|c|c|}
\hline Subfamily & $\mathrm{TCDB} \#^{*}$ & Protein & Function & PDB code & $\begin{array}{c}\text { Resolution } \\
\left(R_{\text {free }}\right)\end{array}$ & Organism & Reference \\
\hline $\begin{array}{l}\text { Oligosaccharide: } \mathrm{H}^{+} \\
\text {symporter (OHS) }\end{array}$ & 2.A.1.5.1 & $\mathrm{LacY}$ & $\begin{array}{c}\text { Lactose: } \mathrm{H}^{+} \\
\text {symport }\end{array}$ & $\begin{array}{l}\text { 1PV6, 1PV7, } \\
\text { 2CFP, 2CFQ, } \\
\text { 2V8N, 2Y5Y, } \\
\text { 4OAA }\end{array}$ & $\begin{array}{c}2.95 \AA \\
(0.298)\end{array}$ & E. coli & {$[15-19]$} \\
\hline $\begin{array}{l}\text { Organophosphate: } \mathrm{Pi} \\
\text { antiporter (OPA) }\end{array}$ & 2.A.1.4.3 & GlpT & $\begin{array}{c}\text { Glycerol-3- } \\
\text { phosphate: } \mathrm{PO}_{4}^{-3} \\
\text { antiporter }\end{array}$ & 1PW4 & $\begin{array}{l}3.30 \AA \\
(0.325)\end{array}$ & E. coli & {$[20]$} \\
\hline $\begin{array}{l}\text { Drug: } \mathrm{H}^{+} \text {antiporter } \\
\text { (DHA1) }\end{array}$ & 2.A.1.2.9 & EmrD & $\begin{array}{l}\text { Multidrug } \\
\text { transporter }\end{array}$ & $2 \mathrm{GFP}$ & $\begin{array}{l}3.50 \AA \\
(0.350)\end{array}$ & E. coli & {$[21]$} \\
\hline $\begin{array}{l}\text { Fucose: } \mathrm{H}^{+} \text {symporter } \\
\text { (FHS) }\end{array}$ & 2.A.1.7.1 & FucP & $\begin{array}{l}L \text {-Fucose: } \mathrm{H}^{+} \\
\text {symporter }\end{array}$ & 307Q, 3O7P & $\begin{array}{c}3.14 \AA \\
(0.273)\end{array}$ & E. coli & {$[22]$} \\
\hline $\begin{array}{l}\mathrm{H}^{+} \text {-dependent } \\
\text { oligopeptide } \\
\text { transporter (POT) }\end{array}$ & 2.A.17 & PepT & $\begin{array}{l}\text { Peptide: } \mathrm{H}^{+} \\
\text {symporter }\end{array}$ & $\begin{array}{l}\text { 4IKV, 4IKW, } \\
\text { 4IKX, 4IKY, } \\
\text { 4IKZ, 4APS, } \\
\text { 2XUT, 4LEP }\end{array}$ & $\begin{array}{l}1.90 \AA \\
(0.216)\end{array}$ & $\begin{array}{c}\text { G. } \\
\text { kaustophilus } \\
\text { St. } \\
\text { thermophiles } \\
\text { Sh. oneidensis }\end{array}$ & [23-26] \\
\hline Sugar porter (SP) & 2.A.1.1.3 & XylE & $\begin{array}{l}\text { D-Xylose: } \mathrm{H}^{+} \\
\text {symporter }\end{array}$ & $\begin{array}{l}\text { 4GBY, 4GBZ, } \\
\text { 4GC0, 4JA3, } \\
\text { 4JA4 }\end{array}$ & $\begin{array}{l}2.60 \AA \\
(0.246)\end{array}$ & E. coli & {$[27,28]$} \\
\hline $\begin{array}{l}\text { Solute carrier family } \\
\text { (SLC22) }\end{array}$ & 2.A.1.9.10 & PiPT & $\mathrm{PO}_{4}^{-3}: \mathrm{H}^{+}$symport & $4 \mathrm{~J} 05$ & $\begin{array}{l}2.90 \AA \\
(0.259)\end{array}$ & P. indica & {$[29]$} \\
\hline Sugar porter (SP) & 2.A.1.1.3 & GlcP & $\begin{array}{l}\text { D-Glucose: } \mathrm{H}^{+} \\
\text {symporter }\end{array}$ & 4LDS & $\begin{array}{l}3.20 \AA \\
(0.341)\end{array}$ & S. epidermidis & {$[30]$} \\
\hline $\begin{array}{l}\text { Nitrate: nitrite porter } \\
\text { (NNP) }\end{array}$ & 2.A.1.8.10 & NarU & $\begin{array}{c}\mathrm{NO}_{3}^{-} / \mathrm{NO}_{2}^{-} \\
\text {exchanger }\end{array}$ & 4IU8, 4IU9 & $\begin{array}{l}3.01 \AA \\
(0.316)\end{array}$ & E. coli & {$[31]$} \\
\hline $\begin{array}{l}\text { Nitrate: nitrite porter } \\
\text { (NNP) }\end{array}$ & 2.A.1.8.1 & NarK & $\begin{array}{c}\mathrm{NO}_{3}^{-} / \mathrm{H}^{+} \\
\text {symporter }(\mathrm{K} 1) ; \\
\mathrm{NO}_{3}^{-} / \mathrm{NO}_{2}^{-} \\
\text {antiporter }(\mathrm{K} 2)\end{array}$ & 4JR9, 4JRE & $\begin{array}{l}2.60 \AA \\
(0.261)\end{array}$ & E. coli & {$[32]$} \\
\hline $\begin{array}{l}\text { Glycoside-pentoside- } \\
\text { hexuronide: cation } \\
\text { symporter }(\mathrm{GPH})\end{array}$ & 2.A.2.1.1 & MelB & $\begin{array}{c}\text { Melibiose: } \mathrm{Na}^{+} \\
\mathrm{Li}^{+}, \text {or } \mathrm{H}^{+}\end{array}$ & $4 \mathrm{M} 64$ & $\begin{array}{c}3.35 \AA \\
(0.359)\end{array}$ & $\begin{array}{c}\text { S. } \\
\text { typhimurium }\end{array}$ & {$[33]$} \\
\hline NRT1/PTR family & 2.A.17.3 & NRT1.1 & $\begin{array}{l}\text { Low affinity } \mathrm{NO}_{3}^{-} \\
\text {transporter }\end{array}$ & $\begin{array}{c}\text { 4CL4, 4CL5, } \\
\mathbf{4 0 H 3}\end{array}$ & $\begin{array}{l}3.25 \AA \\
(0.307)\end{array}$ & A. thaliana & {$[34,35]$} \\
\hline $\begin{array}{l}\text { Solute carrier family } \\
\text { (SLC2A1) }\end{array}$ & 2.A.1.1.28 & GLUT1 & D-Glucose uniport & $4 \mathrm{PYP}$ & $\begin{array}{c}3.17 \AA \\
(0.278) \\
\end{array}$ & Homo sapiens & {$[36]$} \\
\hline
\end{tabular}

${ }^{*} \mathrm{TCDB}$ \# is the transporter classification number obtained from the Transporter Classification Database, http://www.tcdb.org/.

the common structural MFS fold (Figure 2). Most MFS transporters consist of 400-600 amino acyl residues in length, with the core helices arranged into two pseudosymmetrical six-helix bundles [41, 42, 49]. The structure models of MFS transporters reveal that residues postulated to play an important role in catalysis are located at the apex of a deep hydrophilic central cavity formed by two pseudosymmetrical six-helix bundles (Figure 2(a)). The two bundles contain two inverted-topology repeats of three-helix bundles each (Figure 2(c)) [50]. It has been postulated, based on sequence analysis, that each of the two domains may have originated from a gene duplication event [51]. Furthermore, it was demonstrated that inverted-topology repeats encode all the structural information required for the generation of the inward- and outward-facing conformers. Thus, swapping the conformations of the two repeats by comparative modeling indicated that the conversion between states may occur by interchanging the conformations of their inverted-topology repeats $[50]$.

\section{Lactose Permease (LacY)}

The paradigm of the MFS, lactose permease (LacY), is arguably the most intensively studied secondary transporter at present (reviewed in [52-56]). Because LacY and many other MFS transporters were apparently so resistant to traditional means of structural analysis, alternative approaches were developed to approximate the overall 3D fold, as well as local interactions. LacY has been the test bed for the development of most of these methodologies with transport proteins, and they include mapping transmembrane segments by phoA fusions [57], protein insertion into loops and 
deletion analysis [58-60], accessibility/reactivity of natural or uniquely engineered Cys residues to membrane-permeant and impermeant reagents [61], determination of secondary structure by circular dichroism (CD) [62], laser Raman [63], or Fourier transform infrared spectroscopy (FTIR) [64], identifying long-range contacts by suppressor analysis [65, 66], thiol cross-linking [67, 68], excimer fluorescence [69], engineered $\mathrm{Mn}$ (II) binding sites [70], site-directed electron paramagnetic resonance $[71,72]$, or discontinuous $\mathrm{mAb}$ epitope mapping $[73,74]$.

3.1. Transport Modes. Coupling between sugar and $\mathrm{H}^{+}$ translocation is obligatory in LacY. In presence of a $\Delta \widetilde{\mu}_{\mathrm{H}^{+}}$, uphill sugar transport (against its concentration gradient) is coupled to the downhill $\mathrm{H}^{+}$influx (thermodynamically favored movement). In the absence of $\Delta \widetilde{\mu}_{\mathrm{H}^{+}}$, downhill sugar influx (Figure 1(c)) or efflux (Figure 1(d)) will drive uphill $\mathrm{H}^{+}$transport with the generation of $\Delta \widetilde{\mu}_{\mathrm{H}^{+}}$. Besides uphill and downhill transport, LacY catalyzes also two additional translocation modes, exchange and counterflow. For equilibrium exchange, RSO vesicles are preequilibrated with radiolabeled sugar and diluted into medium containing an equimolar concentration of unlabeled sugar. Under saturating conditions, rapid exchange of internal radiolabeled sugar with external unlabeled sugar occurs leading to rapid loss of radiolabeled sugar from the vesicles (Figure 3(a)). Notably, unlike efflux (or influx), exchange is not influenced by $\Delta \psi$ and $\Delta \mathrm{pH}$, and the rate of exchange is unaffected by deuterated buffer (Figure 3(a), right panel) [37, 75]. As it will be discussed in more detail later, the exchange reaction involves only the part of transport cycle where $\mathrm{H}^{+}$binding and release are not involved. In the counterflow experiment, RSO vesicles are preequilibrated with a high concentration of unlabeled lactose (e.g., $10 \mathrm{mM}$ ) and reaction is initiated by dilution into medium containing a lower concentration of radiolabeled lactose (Figure 3(b)). The counterflow reaction is composed of two part reactions, exchange followed by efflux. Due to the much higher rate of exchange, external radiolabeled lactose rapidly replaces the internal cold lactose, which leads to the accumulation of radioactivity by the vesicles by exchange. This process terminates when all of the internal cold lactose has been exchanged with external hot lactose. At this point, efflux predominates (Figure 3(b)) and the radioactivity in the vesicles decreases for two reasons: (i) the concentration gradient (in > out) dissipates and (ii) exchange continues, but the specific activity of the radiolabeled lactose is continuously decreased by the unlabeled lactose that exchanges out of the vesicles. Remarkably, the apparent $K_{m}$ for counterflow is essentially the same as that obtained for $\Delta \widetilde{\mu}_{\mathrm{H}^{+}}$driven uphill transport [75]. Since, imposition of $\Delta \tilde{\mu}_{\mathrm{H}^{+}}, \Delta \Psi$, or $\Delta \mathrm{pH}$ has no effect whatsoever on exchange or counterflow, it is apparent that sugar binding and dissociation drive the alternating access mechanism involved in symport.

3.2. Conformations. Diverse biochemical and biophysical approaches provide consistent evidence that LacY is highly dynamic and that binding of sugar induces widespread conformational changes, increasing the open probability of the periplasmic cavity, with closure of the cytoplasmic cavity in a reciprocal fashion $[55,56,76,77]$. These coordinated conformational transitions represent the basis for the alternating access mechanism. Accordingly, the catalytic cycle of an MFS transporter, LacY, for example, does not involve significant movement of sugar- and $\mathrm{H}^{+}$-binding sites relative to the membrane. Rather, the protein essentially moves around the substrate, reciprocally exposing the binding sites to either side of the membrane (i.e., alternating access in [78]). Although it is generally believed that all MFS symporters operate by an alternating access mechanism, extensive biochemical and spectroscopic evidence is available for LacY only (reviewed in $[55,56])$.

Intuitively, the alternating access mechanism requires at least two conformations: "inward-open," with the substrate binding site open to the cytoplasm, and "outward-open," with the substrate binding site accessible from the periplasmic side. Despite intense efforts, crystals of LacY have not yet been obtained in an outward-open conformation. However, FucP ( $L$-fucose $/ \mathrm{H}^{+}$symporter from $E$. coli), a related MFS member, has been crystallized in the outward-open conformation [22]. The comparative models of outward-open LacY based on the FucP coordinates agree well with models proposed for the outward-open conformer of LacY [50]. Furthermore, biochemical and biophysical studies suggest the existence of additional conformations of LacY. Site-directed alkylation of residues in the cytoplasmic and periplasmic cavity of LacY mutants D68E and D68N reveals a phenotype consistent with a LacY conformer where both cavities are closed to the bulk medium in the presence of sugar [38], constituting the occluded state. In the mutants with replacements of Asp68, active transport is abolished but the mutants retain the ability to bind galactopyranoside. Site-directed alkylation studies show that sugar binding to the Asp68 mutant causes closure of the cytoplasmic cavity, similar to WT LacY; however, strikingly different from WT, opening of the periplasmic cavity upon sugar binding is paralyzed in Asp68 mutants (Figure 4) [38].

Site-directed spin labeling followed by double electronelectron resonance (DEER) measurements $[79,80]$ is highly sensitive to distance changes within $20-60 \AA$ range in proteins, which is compatible with the size of LacY. Nitroxidelabeled paired-Cys replacements on the cytoplasmic or periplasmic sides of LacY at the ends of helices in the $\mathrm{N}$ - and C-terminal helix bundles were used for distance measurements. Multiple distance distributions are typically observed and sugar binding shifts the distance populations towards shorter distances on the cytoplasmic side, whereas longer distances prevail on the periplasmic side in contrast to the substrate free state $[39,72]$. The shortest and longest distances were attributed to the open conformer (longest distance) and closed conformer (shortest distance) on the respective sides of LacY, and the intermediate distances were attributed to the protonated sugar-bound conformer [72]. However, the occurrence of two intermediate distances suggested the presence of two structurally distinct intermediate conformations (Figure 5). Comparative modeling based on X-ray structures in conjunction with analysis of intermediate distances provides further support for the presence of intermediate conformation(s) with the central cavity closed to either 

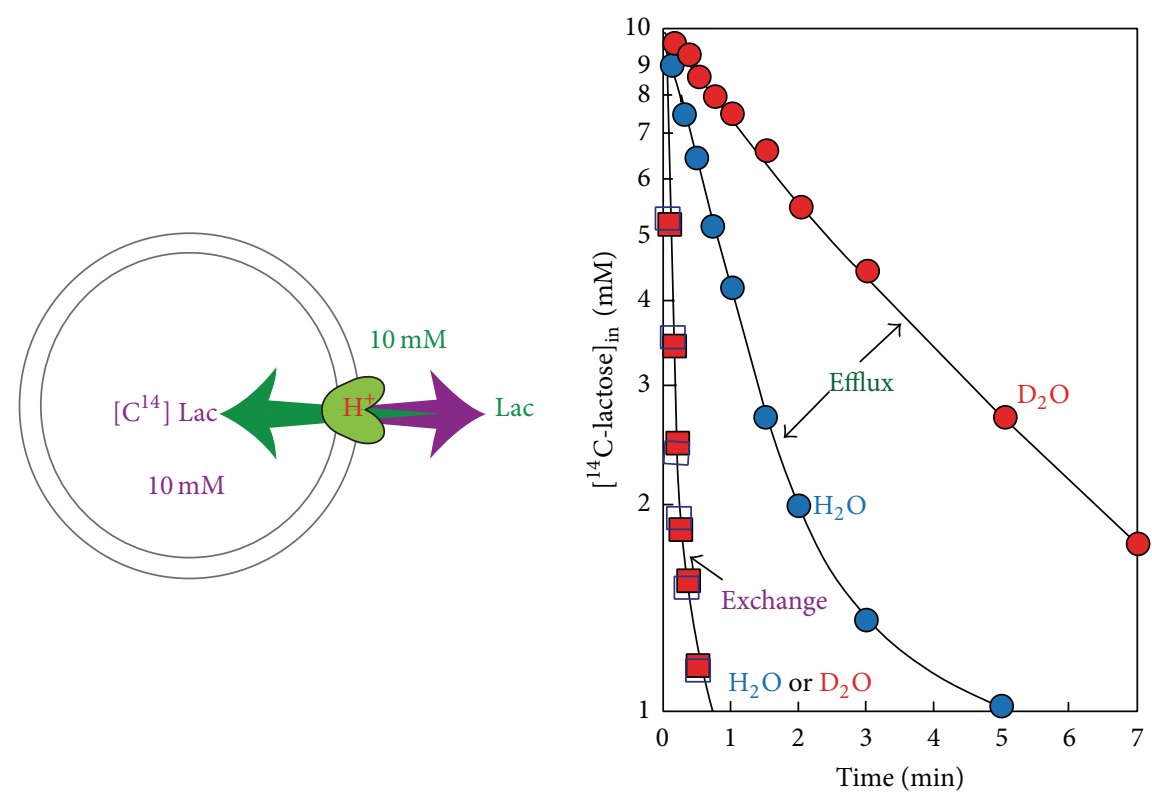

(a) Exchange

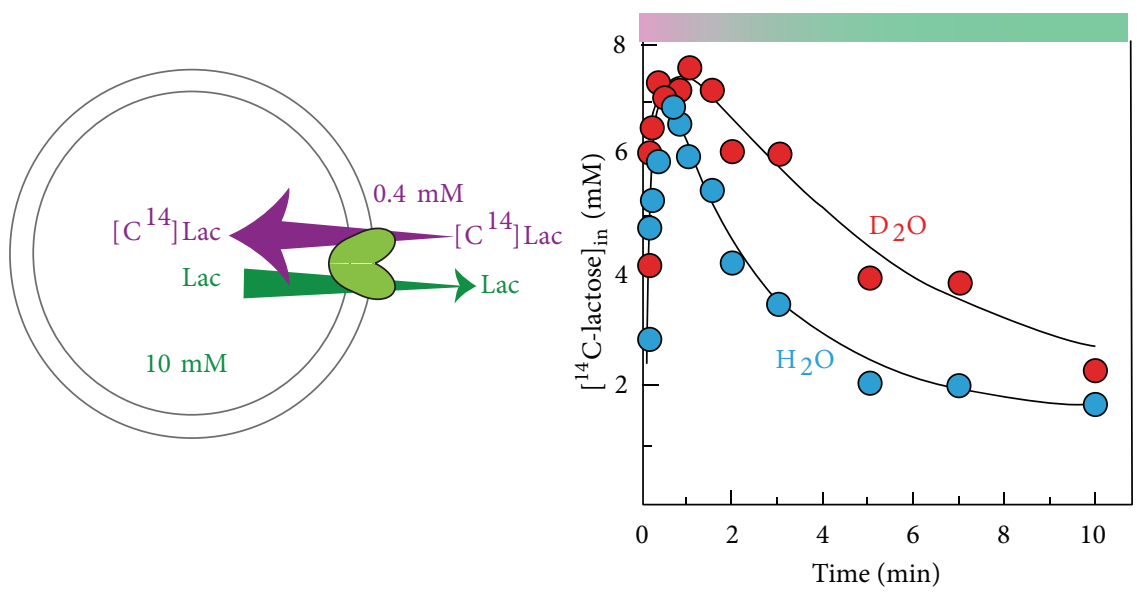

(b) Counterflow

Figure 3: Artificial translocation modes. (a) Schematics of equilibrium exchange: concentrated suspensions of vesicles are equilibrated passively with radioactive lactose. Aliquots are then diluted into buffer with cold lactose (equimolar concentration). Right panel: typical time traces of efflux and exchange showing the effect of deuterium oxide at equivalent $\mathrm{pH}$ and $\mathrm{pD}$ 7.5. Efflux is shown as circles and exchange as squares. Time traces recorded in $\mathrm{H}_{2} \mathrm{O}$ are shown in blue color and data collected in deuterium oxide are shown in red color. (b) Schematics of counterflow experiment: concentrated suspensions of vesicles are equilibrated passively with substrate, and aliquots are diluted into buffer containing radioactively labeled substrate at a much lower concentration. Right panel: typical time traces of counterflow showing the effect of deuterium oxide (color coding is the same as in (a)). The bar on top of the plot indicates the extent of the two part-reactions, exchange (magenta) and efflux (green). The time traces in (a) and (b) are adapted from [37].

side of the membrane [39]. An occurrence analysis of DEER distances that are compatible with the modeled structure revealed that the presence of galactoside does not promote the formation of this LacY conformer, whereas the occurrence of the remaining intermediate distances correlates strongly with the presence of substrate. Therefore, the interspin distances of which the occurrence is enhanced by galactoside binding are attributed to the protonated occluded-intermediate conformation and the distances compatible with the model to an apo intermediate free of sugar [39].
Structural comparison between the inward-open and other conformers of MFS transporters appears to support the rocker-switch model that exploits rigid body movement of the two six-helix domains for alternating access $[20,28$, $81,82]$. However, although $\sim 65 \%$ of the side chains are hydrophobic and buried, $\sim 85 \%$ of the backbone amide protons exchange with deuterium in 10-15 min at room temperature $[83,84]$, with $100 \%$ exchange at elevated, nondenaturing temperatures [85], thereby implying a highly dynamic state of the transporter. Furthermore, the observations that the 


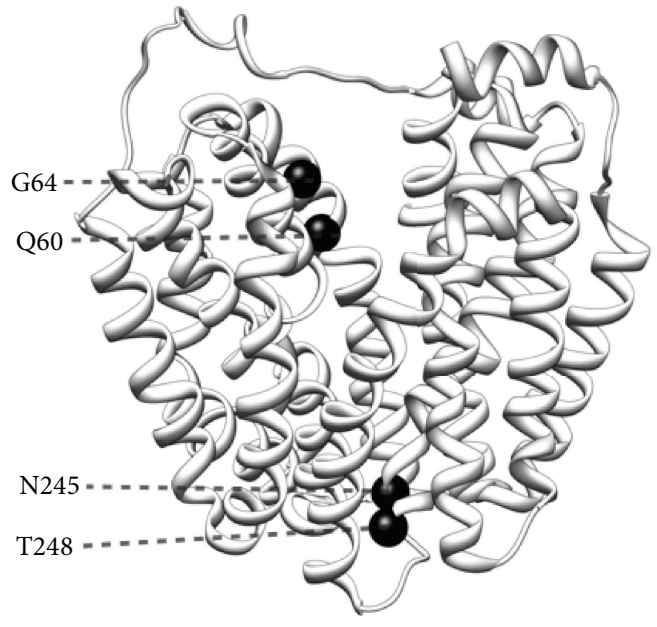

(a)

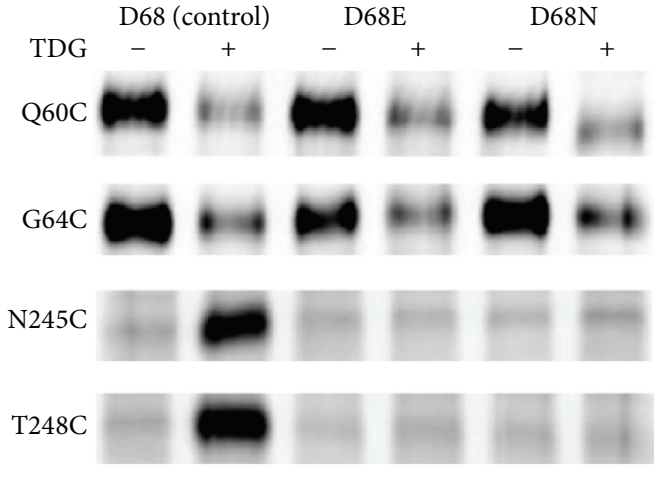

(b)

Figure 4: Site-directed alkylation of LacY mutants. The experiment demonstrates the accessibility of residues in the cytoplasmic or periplasmic cavity. (a) The ribbon structure of LacY (PDBid 2CFQ) with indicated positions of single-Cys replacements shown as black spheres. (b) Fluorescence scans of SDS-PAGE visualizing LacY mutants D68E and D68N, each in a single-Cys background at positions 60, 64,245 , or 248 labeled with tetramethylrhodamine-5-maleimide in the absence or presence of $10 \mathrm{mM}$ TDG. Figure adapted from [38].

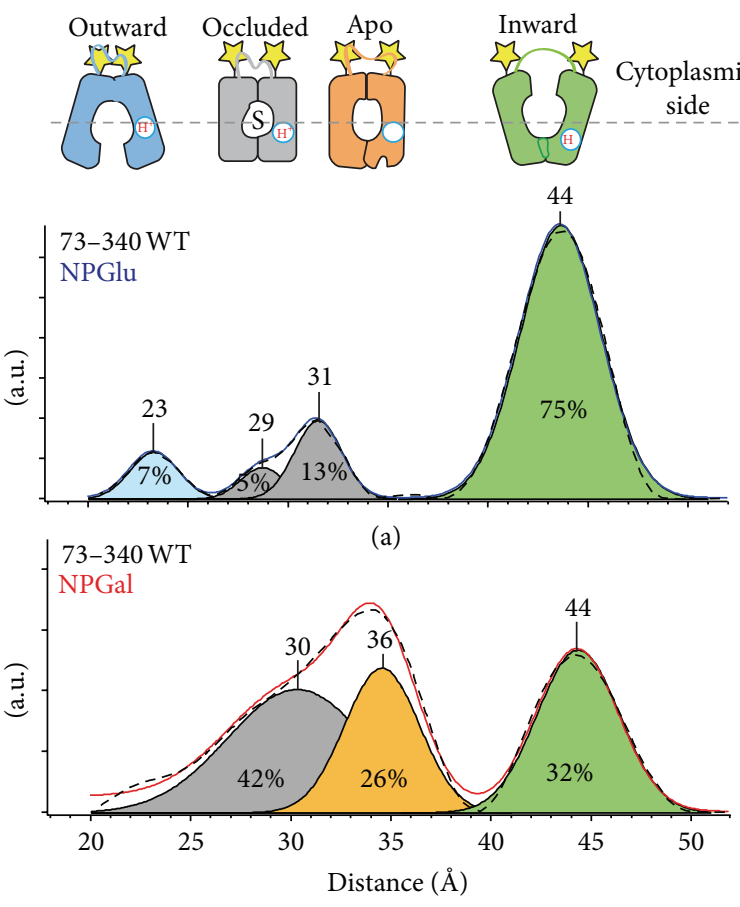

(b)

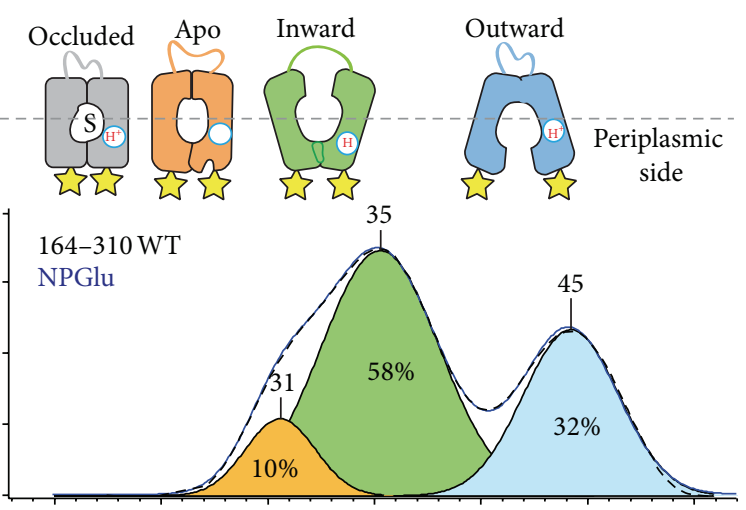

(c)

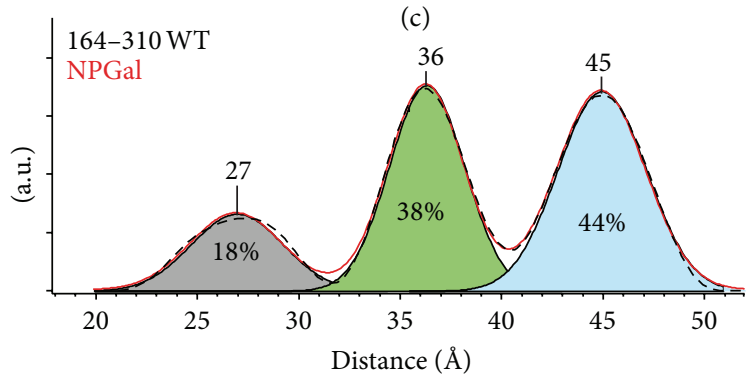

(d)

FIGURE 5: Interspin distance distributions detected with nitroxide-labeled periplasmic Cys pair 164/310 ((a) in absence of a binding sugar and (b) in presence of a binding sugar) and cytoplasmic Cys pair 73/340 ((c) in absence of a binding sugar and (d) in presence of a binding sugar). The top panel shows the respective conformations to which the interspin distances are attributed: blue: outward-facing; gray: occluded intermediate; orange: apo intermediate; and green: inward-facing; the yellow stars indicate the position of the label on LacY relative to the membrane. Distance distributions obtained by Tikhonov regularization of dipolar spectra are shown (solid blue line with no bound sugar; solid red line with bound sugar). Relative distributions of conformational populations are obtained by multi-Gaussian deconvolution. Peak centers, indicated on top of the Gaussian peaks, represent the distance between the nitroxides at these positions (interspin distance). The relative area of the respective Gaussian peaks is indicated (\%) and represents the fraction of each conformational population in the multiGaussian fit (broken black line). The figure is adapted from [39]. 


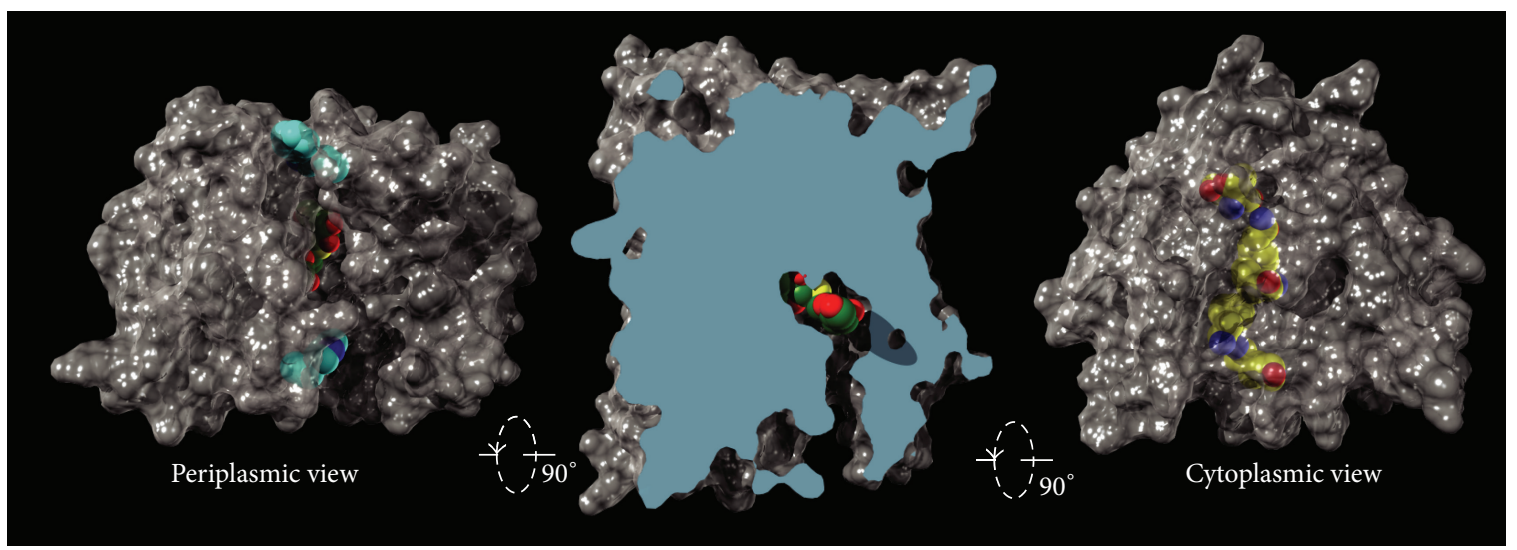

(a)

(b)

(c)

Figure 6: Surface renditions of LacY G46W/G262W (PDBid 4OAA). (a) View from the periplasmic side showing the double-Trp mutantG46W/G262W (cyan sphere model). The TDG molecule is just visible within the protein (sphere model: green: carbon, red: oxygen, and yellow: sulfur). (b) Slab view of the structure seen parallel to the membrane. (c) View from the cytoplasmic side, with the residues that form a zipper-like motif to seal that side portrayed as yellow sphere model (yellow: carbon, red; oxygen, and blue: nitrogen).

transport is blocked when helices within the $\mathrm{N}$ - or Cterminal helix bundles are cross-linked $[38,86,87]$ suggest conformational changes within the two domains. Moreover, crystal structures and theoretical models have been presented for LacY and other MFS transporters in an occluded conformation $[19,24,27,29,39,88,89]$ where a simple concentric rotation of the six-helix domains may be insufficient.

A recent crystallographic model of LacY was obtained in a conformation with an occluded, fully liganded sugar molecule (Figure 6) [19]. This structure was obtained with a double-Trp mutant (G46W/G262W) in which two strategically placed Trp residues replaced Gly residues in each of two Gly-Gly pairs where the N- and C-terminal 6-helix bundles closely interact [90]. The presence of Trp residues at these positions causes the periplasmic cavity, which is closed in absence of substrate, to open. But when binding occurs and the mutant attempts to transition into an occluded state, it cannot do so completely because the bulky Trp residues at positions 46 and 262 block complete closure. Thus, the mutant is able to bind sugar and initiate transition into an occluded state, which it cannot complete. Therefore, the mutant is completely unable to catalyze transport of any type across the membrane [90]. The conformation of the high-affinity sugar ( $\beta$-D-galactopyranosyl-1- thio- $\beta$ D-galactopyranoside, TDG) molecule is clearly defined in central cavity and allows assignment of likely hydrogen bond interactions with the protein (Figure 7) [19].

3.3. The Active Site. Early studies with LacY show that galactoside transport is inactivated by $\mathrm{N}$-ethylmaleimide (NEM) and that the protein can be selectively labeled with radioactive NEM by substrate protection against alkylation [91]. Based on the findings, it was suggested that a Cys residue is in the active site of LacY, and Bieseler et al. demonstrated that the substrate-protected residue is Cys148 [92]. Surprisingly, however, site-directed mutagenesis studies demonstrated that replacing Cys148 with either Ser or Gly abolishes neither lactose accumulation nor sugar binding [93,
94]. These studies led to the systematic mutagenesis of each of the remaining seven native Cys residues, showing that none is essential for transport, and to the ultimate construction of a functional LacY mutant devoid of Cys residues. To determine which residues in LacY play an obligatory role in transport and to create a library of mutants with a single-Cys residue at each position of LacY for structure/function studies, each residue in Cys-less LacY was replaced individually with Cys or other side chains [95]. The great majority of the single-Cys mutants are expressed normally and catalyze accumulation of lactose against a significant concentration gradient, thereby demonstrating that Cys replacement at most positions does not lead to severe retardation of the structure of LacY or in the symport mechanism. Fewer than 10 residues are irreplaceable for active lactose transport, while Cys substitution of only another $\sim 82$ residues inhibits the activity of the steadystate level of accumulation by 50-80\% [95]. Crystallographic models of LacY [15] and GlpT [20] reveal what is now known as the typical MFS fold (Figure 2), as well as the location of most of the irreplaceable residues at the approximate middle of the LacY molecule.

The X-ray structure of the sugar-occluded LacY conformer [19] is highly consistent with mutational, functional, and spectroscopic results (reviewed in $[52,54,55]$ ). An aromatic side chain at the position 151 in LacY at the apex of the inward-facing hydrophilic cavity is an absolute requirement for binding and transport. The galactopyranosyl ring and the indole ring of Trp151 (helix V) form the primary hydrophobic interaction between LacY and the galactopyranosyl substrates [96]. Trp151 can be replaced with Tyr or Phe with progressively decreased affinity, but absence of an aromatic side chain at this position completely abolishes binding and transport. In addition, the red shift in the phosphorescence spectrum of single-Trp151 LacY observed upon sugar binding [97] is best explained by the apposition of the galactopyranosyl and indole rings (Figure 7). Cys substitution, sitedirected alkylation, and direct binding assays indicate that Arg144 (helix V) and Glu126 (helix IV) are irreplaceable 


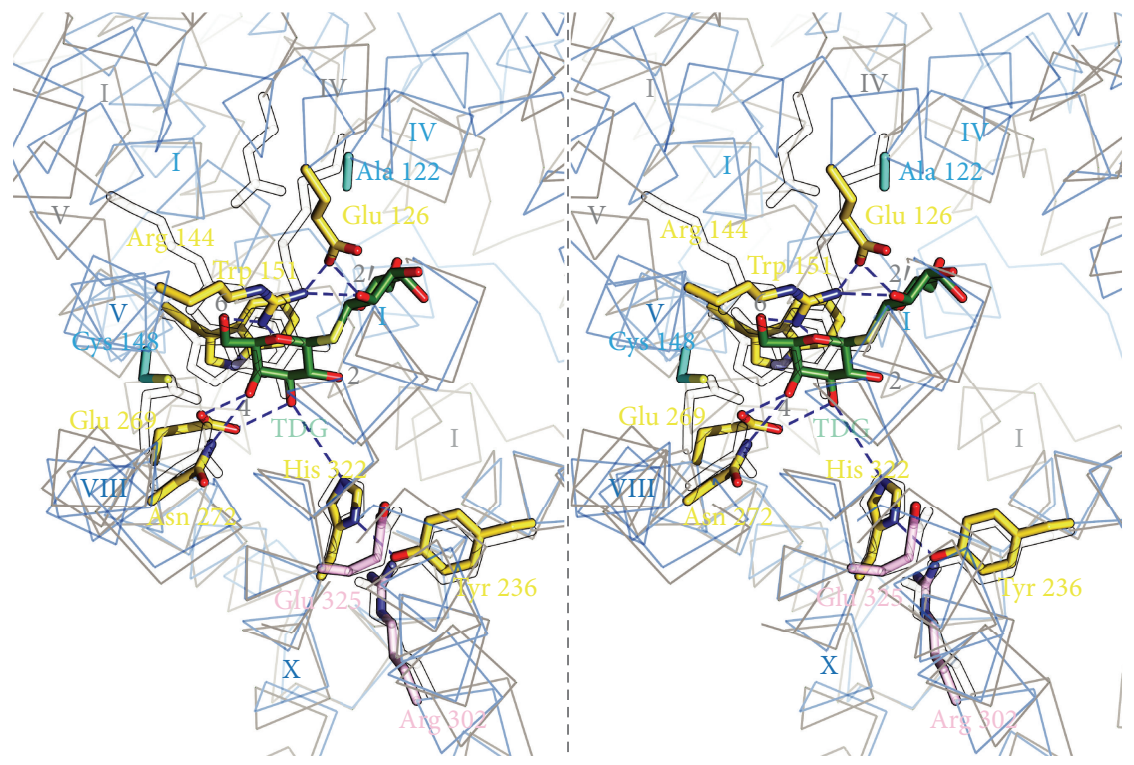

Figure 7: Cytoplasmic view of sugar-binding site in LacY (stereo view). LacY structure with bound sugar (PDBid: 4OAA, blue trace, and colored side chains) is superposed on the LacY structure with covalently bound MTS-Gal (PDBid: 2Y5Y, gray trace and transparent side chains). TDG is shown as green sticks, and side chains forming hydrogen bonds with TDG are in yellow. Broken lines represent likely hydrogen bonds. Ala122 and Cys148, which are close to TDG but do not make direct contact, are shown in light blue. Glu325 and Arg302 are shown in pink color. Roman numbers indicate the helix number.

for substrate binding [98-100]. These side chains interact with $\mathrm{C} 6-\mathrm{OH}, \mathrm{O} 5$, and $\mathrm{C}^{\prime}-\mathrm{OH}$ of the galactopyranosyl ring [19], and, interestingly, helices $\mathrm{V}$ and IV display a significant tilt compared to the inward-open conformation of LacY (Figure 7). Furthermore, the irreplaceable residue, Glu269 (helix VIII), which cannot even be replaced with Asp without markedly decreasing affinity [101], is the acceptor of hydrogen bonds from the $\mathrm{C} 4-\mathrm{OH}$ and $\mathrm{C} 3-\mathrm{OH}$ groups of the galactopyranosyl ring, making it a likely candidate for a primary role in specificity. Galactose and glucose, as well as the respective galactosides and glucosides, differ only in the orientation of the $\mathrm{C} 4-\mathrm{OH}$ of the galactopyranosyl ring, and LacY has little or no affinity for glucose or glucopyranosides. Asn272 is also within a $\mathrm{H}^{+}$-bounding distance to $\mathrm{C} 4-\mathrm{OH}$, and replacing Asn272 with almost anything but Gln either markedly decreases affinity for substrate (i.e., high $K_{D}$ ) or abolishes binding altogether [102]. Although the specificity of $\mathrm{LacY}$ is strongly directed toward the $\mathrm{C} 4-\mathrm{OH}$ of the galactopyranosyl ring, other $\mathrm{OH}$ groups also are important in the following order: $\mathrm{C} 4-\mathrm{OH} \gg \mathrm{C} 6-\mathrm{OH}>\mathrm{C} 3-\mathrm{OH}>\mathrm{C} 2-$ $\mathrm{OH}[103,104]$. As observed in the structure, His322 (helix $\mathrm{X}$ ), which is held in place by Tyr236 (helix VII), acts as a hydrogen bond donor between the $\delta \mathrm{NH}$ of the imidazole ring and the $\mathrm{C} 3-\mathrm{OH}$ of TDG. All replacements for His322 result in a protein with markedly decreased binding affinity and little or no transport activity $[101,105,106]$, thereby demonstrating that His322 is an essential constituent of the sugar-binding site.

Neutral replacements for Glu325 (helix X) yield mutants that are defective in all transport reactions that involve lactose $/ \mathrm{H}^{+}$symport but catalyze equilibrium exchange and/or counterflow as well as or better than WT [107-109], and replacement of Arg302 (helix IX) with Ser or Ala results in a similar phenotype [109]. Since neutral replacements for Glu325 bind ligand normally and mutants R302K and R302A exhibit only a modest decrease in affinity [101], the two residues are probably exclusively involved in $\mathrm{H}^{+}$ translocation. The two side chains flank the His322-Tyr236 pair on the cyto- and periplasmic side, respectively (Figures 2(a) and 7), but neither residue is sufficiently close to the bound sugar to make direct contact. It is noteworthy that Glu325 is embedded in a hydrophobic milieu formed by Met299 and Ala295 (helix IX), Leu329 (helix X), and Tyr236 (helix VII), supporting the notion that Glu325 is protonated in this conformation [54]. Since Glu325 and Arg302 exhibit similar phenotypes, it was suggested that Arg302 drives deprotonation of Glu325 [109]. Consistent with this idea, LacY with two Cys residues at positions 302 and 325 exhibits excimer fluorescence [69], and with two His replacements, a $\mathrm{Mn}(\mathrm{II})$ binding site is observed [110]. However, in the available X-ray structures, the side chain of Arg302 is 7 $\AA$ removed from Glu325 and separated by Tyr236. Therefore, a conformational rearrangement must occur for $\operatorname{Arg} 302$ to come sufficiently close to Glu325 to cause deprotonation [39].

In addition to the residues that make direct contact with bound galactoside, Cys148 (helix V), mentioned above in the context of substrate protection against alkylation (reviewed in [54]), is close to bounding TDG but is not sufficiently close to making contact (Figure 7). Similarly, replacement of Ala122 (helix IV) with bulky side chains or alkylation of A122C with bulky thiol reagents renders LacY specific for transport of the monosaccharide galactose, and disaccharide binding and transport are blocked [111]. However, Ala122 also does not make a direct contact with TDG (Figure 7). Clearly, 
the properties of Cys148 and Ala122 mutants with respect to sugar binding are due to steric effects.

3.4. Additional Important Residues. The overall structures of LacY and other MFS members show that the transporters consist of irregular, highly kinked transmembrane helices (see PDB files in Table 1), and residues that introduce structural irregularity (i.e., Pro and Gly) align in the kinked regions of the helices $[39,50]$. However, most replacements of the twelve Pro residues in LacY with Gly, Ala, or Leu and deletion of Pro403 and Pro405 in the C-terminal tail are well tolerated [112]. With the exception of Pro28, it was found that, primarily, the hydrophobicity and/or size of the side chain is significant for efficient transport function. Similar studies have been performed on the Gly residues in LacY [113]. The results indicate that although none of the Gly residues in LacY is mandatory for activity, the bulk of the side chain at positions 64,115 , and 147 , rather than conformational flexibility at these positions per se, is particularly important. Even though Pro is more frequent near a kink, only $33 \%$ of transmembrane bends actually have a Pro residue within one helical turn [114]. It is likely that an existing kink is stabilized at a distant position by restraining the helix in a kinked form. Thus, the irregularity in the helices in LacY is not due to the presence of a Pro or Gly residue but on the hydrophobicity and/or size of the side chain at this position or interactions with other helices in the 3D structure.

Essential to obtaining the first X-ray structure of LacY was the use of a mutant in which Cys154 (helix V) is replaced with Gly [15]. C154G LacY mutant exhibits fewer liganddependent conformational changes than observed with WT LacY and catalyzes little or no transport but binds ligand with high affinity $[72,115,116]$. The X-ray structure demonstrates that helix V crosses helix I in the approximate middle of the membrane in such a manner that Cys154 lies close to Gly24 (helix I). Therefore, it seems likely that the replacement of Cys154 with Gly leads to tighter packing between helices I and $\mathrm{V}$, thereby restricting important conformational flexibility between the two helices. Consistently, replacement of Gly24 with Cys in the C154G mutant dramatically rescues transport activity, and the mutant exhibits properties similar to WT LacY with respect to substrate binding [115]. However, it is puzzling that Cys is the only replacement for Gly24 that exhibits significant ability to rescue transport activity in mutant C154G.

Other important positions, although not directly associated with sugar or $\mathrm{H}^{+}$binding/transport, are positions promoting structural integrity (i.e., charged pairs). The Asp237 (helix VII)/Lys358 (helix XI) charge pair, which is important for membrane insertion and stability of LacY, is a genuine salt bridge. Thus, Asp237 can be replaced with Glu, carboxymethyl-Cys, or sulfonylethylthio-Cys with Lys or Arg at position 358, and good activity is retained. Similarly, LacY tolerates replacement of Lys358 with Arg or ammoniumethylthio-Cys with Asp or Glu at position 237. Remarkably, LacY with Lys, Arg, or ammoniumethylthio-Cys in place of Asp237 is highly active when Lys358 is replaced with Asp or Glu, thereby demonstrating that the polarity of the charge interaction can be reversed without loss of activity [117, 118]. In contrast, the Asp68 (helix II)/Lys131 (helix IV) charge pair, which is involved with the coordinated opening and closing of the cytoplasmic and periplasmic cavities, exhibits marked sensitivity to changes in the nature or polarity of the side chains. Even a short extension of Asp residue by replacement with Glu inactivates transport and activity is not rescued by replacing Lys131 with Arg nor will this pair tolerate interchange [38].

Similarly, with the Asp240 (helix VII)/Lys319 (helix X) charge pair, single replacements of either residue with Ala completely impair active transport [119], and replacement of Asp240 with Glu abolishes lactose transport. Replacement of Lys319 with Arg is tolerated, but the steady-state level of lactose accumulation is significantly decreased [118]. Also, this charge pair cannot be reversed by interchanging the residues [118]. Unlike conservative replacements, neutral replacements for Lys319 result in high-affinity galactoside binding over a wide $\mathrm{pH}$ range, which is homologous to Glu325 neutral replacements [101]. However, neutral replacements for Asp240 (A, N) significantly lower the affinity to sugar and attenuate the $\mathrm{pH}$ dependence of binding. Although the D240E mutant does not support active lactose uptake, permease with carboxymethyl-Cys at this position (D240C) exhibits significant activity when paired with the K319R mutant [118]. Interestingly, sulfonylethylthio-Cys substitution for Asp240 also results in significant transport activity. LacY with Arg or ammonioethylthio-Cys in place of Lys319 exhibits high activity with Asp240 as the negative counterion, but no lactose transport is observed when either of these modifications is paired with D240E mutation [118]. Moreover, mutant K319L or K319Q displays some of the qualities described for Glu325 mutants with neutral replacements [108], in particular, the selective inactivation of lactose active transport or efflux without any significant effect on exchange [120] or affinity for sugar [101, 121]. Hence, it seemed logical that the Asp240/Lys319 charge pair might be involved in $\mathrm{H}^{+}$translocation [101]. However, since the double mutants D240C/K319C and D240C/K319R display significant ability to catalyze lactose accumulation against a concentration gradient, this conjecture is unlikely $[87,118]$. Notably, cross-linking of the D240C/K319C mutant abolishes lactose transport, suggesting that the charge pair may play a role in stabilizing the conformation of the active site [87].

\section{An Overall Mechanism for Coupling in LacY}

Converging lines of evidence demonstrate that LacY functions by an alternating access mechanism (reviewed in [55, 56]). Essential for the mechanism is that the galactosidebinding site in LacY is located in the approximate middle of the molecule [15] where it can become accessible to both sides of the membrane by reciprocal opening and closing of periplasmic and cytoplasmic hydrophilic cavities. Binding of the sugar induces widespread conformational transitions in LacY, increasing the open probability of a hydrophilic cleft on the periplasmic side of the molecule with closure of the cytoplasmic cavity in reciprocal fashion [77]. Alkylation of Cys side chains located near or within the inwardfacing hydrophilic cavity by radiolabeled NEM or fluorescent 


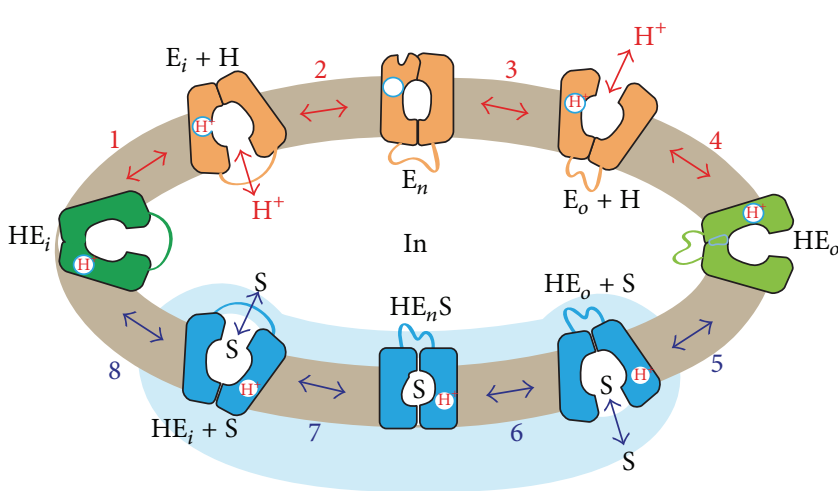

(a)

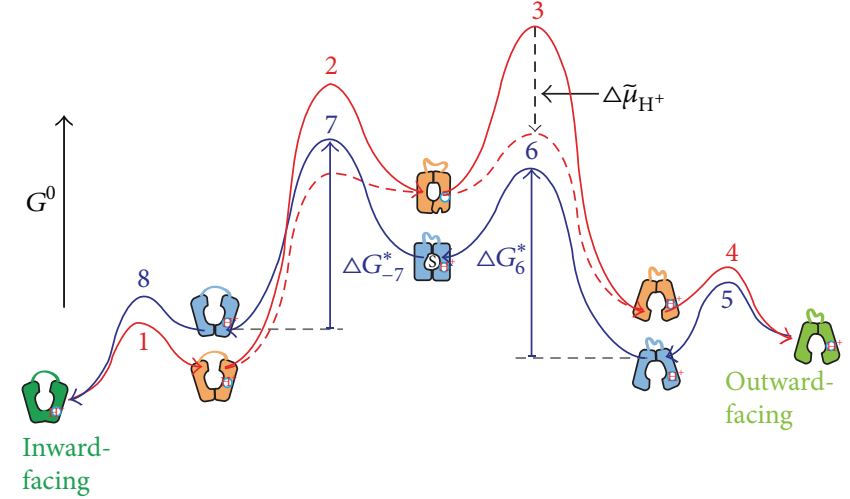

(b)

FiguRE 8: Transport cycle of LacY. (a) Overview of the postulated steps in the transport model. Inward-facing (green) and outward-facing (blue) conformations are separated by the apo-intermediate conformations (orange) or by the occluded-intermediate conformations (gray). Steps are numbered consecutively: 1: opening of the $\mathrm{H}^{+}$site; 2 : deprotonation to inside and reorientation to the apo intermediate with a central cavity closed to either side of the membrane; 3: opening of the outward-facing cavity and reprotonation from the outside; 4 : formation of outward-open, substrate-free conformation; 5 and 6: substrate binding and induced fit to the occluded conformation; 7: opening of the inwardfacing cavity and release of the sugar; and 8: formation of the protonated, substrate-free conformation. The blue-shaded area demarcates the equilibrium-exchange reaction. (b) Hypothetical energy profile for the transport cycle. Conformations shown in (a) are translated into relative energy states (indicated by the icons of conformations defined in (a) with the cytoplasmic side of the symporter facing up). The schemes are cycles read by following the arrowheads. The red part of the cycle represents the transitions between Steps 1 and 4 of the empty pathway. The blue line corresponds to Steps 5-8 for net sugar transporting steps and for the exchange reaction. The hypothetical effect of an imposed $\Delta \widetilde{\mu}_{\mathrm{H}^{+}}$ is shown as a broken black vertical arrow, and the broken red lines show the resulting energy profile. The figure is modified from [39].

tetramethylrhodamine-5-maleimide (TMRM), which are used to study reactivity/accessibility of single-Cys residues introduced into Cys-less LacY, shows markedly decreased reactivity upon galactoside binding. Alternatively, engineered Cys replacements located near or within the periplasmic cavity exhibit reciprocal galactoside-dependent increases in reactivity/accessibility [122]. As proposed initially by Mitchell [13], transport involves movement of LacY around the bound galactoside, allowing alternating access of binding sites to either side of the membrane.

More than three decades ago, an ordered kinetic scheme for lactose $/ \mathrm{H}^{+}$symport by LacY was suggested [75], and the experimental evidence has been refined ever since (Figure 8(a)) [52, 54, 55]. Importantly, the observations that exchange and counterflow are independent of $\mathrm{pH}$ when the lactose concentration is saturating and the rate is not altered by $\mathrm{D}_{2} \mathrm{O}$ [75] together with the behavior of neutralreplacement mutants for Glu325 which catalyze exchange and counterflow at least as well as the WT but do not catalyze any reactions which involves net $\mathrm{H}^{+}$transport [108] lead to the conclusion that an ordered kinetic mechanism is operative in the transport cycle of $\mathrm{LacY}$. The $\mathrm{H}^{+}$binds prior to the galactoside, and the sugar is released on the other side of the membrane before the $\mathrm{H}^{+}$(Figure 8(a)). The notion that LacY must be protonated to bind sugar is consistent with the $\mathrm{pK}_{a}$ for sugar binding being 10.5 [101], as well as other findings [121].

Numerous experimental observations [39, 72, 122, 123] suggest that the transport sequence in $\mathrm{LacY}$ is initiated from the inward-open conformation (Figure 8(a), Step $1, \mathrm{HE}_{i}$ ). In the absence of sugar, the periplasmic side of LacY in the native bacterial membrane is closed, and galactoside binding increases the opening probability of the periplasmic cavity with closing of the inward-open cavity $[68,122]$. Moreover, the $12 \AA$ distance between Trp151 and nitrophenyl moiety at the anomeric position of the galactosyl moiety of bound p-nitrophenyl- $\alpha$-D-galactopyranoside (NPG) results in fluorescence resonance energy transfer (FRET), which is used to determine substrate binding and stopped-flow rates of binding [124]. A decrease of Trp fluorescence resulting from Trp151 * NPG FRET is observed with detergent solubilized $\mathrm{LacY}$ as well as with $\mathrm{LacY}$ reconstituted into proteoliposomes, although the pre-steady-state kinetics of NPG binding to the solubilized LacY differs significantly from that observed with reconstituted LacY. Rates of sugar binding vary linearly with substrate concentration in solubilized LacY, since the binding site is accessible from the inward-facing cavity. However, in reconstituted $\mathrm{LacY}$, where $\mathrm{LacY}$ is inserted unidirectionally with the periplasmic side facing the medium, sugar binding rates are independent of galactoside concentration [125]. This finding suggests that opening of the periplasmic cavity is the limiting step for sugar binding with reconstituted LacY (Figure 8(a), Steps 2 and 3).

4.1. $\mathrm{H}^{+}$Translocation. Current evidence indicates strongly that $\mathrm{LacY}$ is protonated prior to ligand binding $[126,127]$. Thus, in addition to the evidence for the kinetic scheme discussed above (Figure 8), galactoside binding to $\mathrm{LacY}$ as a function of $\mathrm{pH}$ exhibits a $\mathrm{pK}_{a}$ of $\sim 10.5$ [127]. Thus, in the physiological $\mathrm{pH}$ range, LacY clearly has a very high probability of being protonated. Remarkably, neutral replacement of Glu325 abolishes the $\mathrm{pK}_{a}$, and high-affinity binding 
is observed up to $\mathrm{pH} 11$ [101]. Thus, it is apparent that a negative charge at position 325 (i.e., unprotonated Glu325) abrogates binding, and recent studies (Madej, unpublished observations) show that a positive charge also has the same effect. Furthermore, it has been shown directly [121] that addition of TDG to a concentrated solution of purified, detergent solubilized LacY induces no change in $\mathrm{pH}$, while a positive control with the antiporter EmrE (provided by S. Schuldiner, Hebrew University, Jerusalem) under identical conditions releases $1 \mathrm{H}^{+} / \mathrm{mol}$ EmrE upon addition of tetraphenylphosphonium [128].

Although LacY must be protonated prior to galactoside binding, which clearly represents an important part of the coupling mechanism, it is difficult to study the mechanism of $\mathrm{H}^{+}$translocation. However, in addition to $\Delta \widetilde{\mu}_{\mathrm{H}^{+}}$-driven active transport, LacY catalyzes other modes of translocation that are important for studying coupled $\mathrm{H}^{+}$transport. Because individual steps in the overall transport cycle cannot be delineated by studying $\Delta \widetilde{\mu}_{\mathrm{H}^{+}}$-driven active transport, carriermediated efflux down a concentration gradient, equilibrium exchange, and entrance counterflow are used to probe the mechanism $[75,129]$. Efflux, exchange, and counterflow with WT LacY are explained by the kinetic scheme shown in Figure 3.

Many enzyme reactions involve $\mathrm{H}^{+}$transfer in the ratelimiting step, and, as a consequence, these reactions may exhibit a solvent isotope effect when studied in deuterium oxide $\left(\mathrm{D}_{2} \mathrm{O}\right)$. In brief, such reactions proceed slower in $\mathrm{D}_{2} \mathrm{O}$ because of differences in the zero-point stretch vibrations of bonds to protium relative to deuterium [130, 131]. With right-side-out (RSO) vesicles or proteoliposomes reconstituted with purified LacY, 3-4-fold inhibition of the rate of $\mathrm{H}^{+}$-coupled downhill lactose efflux is observed in $\mathrm{D}_{2} \mathrm{O}$ from $\mathrm{pH} 5.5$ to 7.5 , with no effect on $\Delta \tilde{\mu}_{\mathrm{H}^{+}}$-driven active transport, exchange, counterflow, or affinity for sugar [37, $129,132]$. These observations and others indicate that reactions involved in protonation or deprotonation are not rate determining for $\Delta \tilde{\mu}_{\mathrm{H}^{+}}$-driven active transport, exchange, or counterflow, while deprotonation is rate limiting when a lactose concentration gradient drives uphill $\mathrm{H}^{+}$translocation (Figures 1(b) and 1(c)). This conclusion also receives strong support from solid supported membrane electrophysiology with proteoliposomes reconstituted with purified LacY, as well as some of the mutants described above [133-135].

Glu325 is the major if not the only residue directly involved in $\mathrm{H}^{+}$transport in LacY [132]. Although earlier studies suggested by implication that Tyr236, Glu269, and His322 may be involved in $\mathrm{H}^{+}$transport, it is now apparent that these side chains are clearly ligands to the galactopyranoside ring [19]. Although mutation of these residues causes the periplasmic side of LacY to open spontaneously [136], there is no direct evidence that they are involved in $\mathrm{H}^{+}$ translocation, although it is conceivable that one or more may play a dual role in the transport mechanism. It is also notable that every residue in LacY has been subjected to site-directed mutagenesis, and, with the exception of the irreplaceable residues described, each of which has a known function, there are no other side chains in the protein which are absolutely required for lactose $/ \mathrm{H}^{+}$symport.
Molecular dynamics (MD) simulations indicate that the deprotonation of Glu325 causes closing of the internal cavity and reciprocal opening of the periplasmic cavity [77, 137]. All crystallographic models of LacY presented so far show Glu325 embedded in a hydrophobic milieu indicating that it is protonated (Table 1). Kinetic considerations and DEER measurements suggest that an occluded intermediate with an empty substrate binding-site, the apo intermediate, (Figure 8(a), conformer $E_{n}$ ) is involved in the conformational change of apo-LacY from the inward-open to the outwardopen conformer [39]. Arg302 may approximate Glu325 to cause deprotonation by decreasing the $\mathrm{pK}_{a}$ of the protonated carboxyl group $[39,69,109,110]$, and reprotonation of the apo intermediate likely occurs stochastically from the side of the membrane with higher proton activity.

It is important to realize that a $\Delta \tilde{\mu}_{\mathrm{H}^{+}}$has no effect on the substrate affinity of LacY; the $K_{D}$ of sugar binding is the same from both sides of the membrane [138]. Therefore, the widely held notion that active transport involves a change in affinity on opposite sides of the membrane is not valid, at least for sugar binding in LacY. However, either component of $\Delta \widetilde{\mu}_{\mathrm{H}^{+}}$causes the $K_{m}$ to decrease 50-100-fold with little or no change in $V_{\max }$ [139]. The decrease of $K_{m}$ in presence of a $\Delta \widetilde{\mu}_{\mathrm{H}^{+}}$might be due to a lowering of the activation energy for deprotonating and/or reprotonating steps (Figure 8(b), Steps 2 and 3, black arrow) resulting in faster recycling of the open cavity and an increase in its effective concentration (Figure 8(a), $\mathrm{HE}_{o}$ ).

4.2. Sugar Translocation. The X-ray structure of sugaroccluded LacY [19] confirms more than two decades of biochemical and spectroscopic findings [55]. The stoichiometry of sugar binding is 1 galactoside/LacY. Five irreplaceable LacY residues that ligate the galactoside directly (Arg144, Trp151, Glu269, Asn272, and His322) and Glu126 and Tyr236 that stabilize Arg144 and His322, respectively, form the galactoside-binding site in the middle of the protein (Figure 7). Mutation of any of these residues either abolishes completely or severely decreases affinity [101]. Remarkably, unlike the sugar-occluded structure, the structure of LacY with covalently bound methanethiosulfonylgalactopyranoside (Figure 7, gray trace and transparent side chains), where only Trp151 and Glu269 make contact with the galactoside, is in an inward-open conformation [16]. Thus, the sugar must be fully ligated for LacY to transition into the occluded state. The translocation of the sugar probably begins with the interaction between the indole ring of Trp151 and the galactopyranosyl ring providing the primary hydrophobic interaction (Figure 8(a), $\mathrm{HE}_{o}+\mathrm{S}$ ) [96]. As with enzymes, the substrate is not optimally bound in the open state, but, unlike enzymes, the unchanged substrate induces a major reorganization in the binding site to induce an optimum fit (Figure 8(a) Step 6; compare structure superposition in Figure 7) $[16,19]$.

It has been postulated that sugar binding lowers the activation energy barrier for the transition between the inwardopen and outward-open conformers of LacY [39]. Accordingly, increased substrate-protein interaction in the substrateoccluding state provides the binding energy to compensate 
for the energy costs of the concomitant reconfiguration of the transporter (Figure $8(\mathrm{~b}), \Delta G_{6}^{*}$ and $\Delta G_{-7}^{*}$ ). Consequently, galactoside binding permits LacY to overcome energy barriers separating the inward- and outward-open conformers. Results obtained employing dynamic single-molecule force spectroscopy support and expand this hypothesis by providing quantitative insights into kinetic, energetic, and mechanical properties of the sugar-free and sugar-bound states of LacY [116]. Sugar liganding induces a dramatic change in structural properties of the N-terminal 6-helix bundle leading to a global increase in conformational flexibility. Similarly, isothermal titration calorimetry reveals that galactoside binding to WT LacY is predominately entropic, indicating multiple ligand-bound conformational states [140]. In other words, substrate binding involves induced fit, which leads to the expansion of conformational space. In view of these considerations and the direct observation that $\Delta \widetilde{\mu}_{\mathrm{H}^{+}}$has no effect whatsoever on equilibrium exchange or counterflow, the conclusion emerges that galactoside binding and dissociationnot $\Delta \widetilde{\mu}_{\mathrm{H}^{+}}$-are the driving force for the alternating access mechanism (Figures 8(a) and 8(b), Steps 6 and 7). With galactoside dissociation (Figure 8(a), conformer $\mathrm{HE}_{i}+\mathrm{S}$ ), the transporter returns to a more static conformation [116]. The initiation of another round of transport (Figure 8(a), step 2) may result from the deprotonation of the conformer where Arg302 (helix IX) approximates Glu325, although reversible steps are also possible (Figures 8(a) and 8(b), Steps 8 and 1).

Lactose $/ \mathrm{H}^{+}$symport in the active transport, (Figure 1(b); against the lactose concentration gradient-uphill) or efflux or influx, (Figures 3(a) and 3(b); thermodynamically favored movement with lactose concentration gradient-downhill) energetic modes are precisely the same reaction (Figure 8(a)). The difference is in the rate-limiting step. For lactose downhill symport, deprotonation is rate-limiting; for uphill transport, deprotonation is no longer limiting, and either dissociation of sugar or a conformational change that leads to deprotonation becomes limiting $[37,75]$. It appears that coupling between sugar and $\mathrm{H}^{+}$translocation is solely energetic; the transporter (e.g., LacY) transduces the potential energy stored in the concentration gradient of one substrate (e.g., $\mathrm{H}^{+}$) to facilitate the accumulation of another substrate (e.g., galactoside) against its concentration gradient. Accordingly, the hypothetical energy landscapes for the transport cycle in which the occluded intermediates occupy higher energy levels relative to a comparatively facile energy profile between the open conformers are the conformationally dynamic species [116]. The higher energy occluded intermediates tend to relax to lower-energy open conformations (Figure 8(b)). Such energetic disposition, where high-energy barriers separate inward- and outward-open conformers contacting the phases with a potential energy difference, indicates that the transport reaction is thermodynamically driven but controlled kinetically. In other words, $\mathrm{LacY}$ can only conduct a $\mathrm{H}^{+}$-flow down its concentration gradient when a bound galactoside is present to facilitate the conformational transition from, for example, outward-open to inward-open conformation (Figure 8). Notably, the $K_{D}$ for sugar does not change, but the affinity for $\mathrm{H}^{+}$(i.e., the $\mathrm{pK}_{a}$ of Glu325) decreases markedly once the galactoside dissociates.

\section{Mix-and-Match Evolution in MFS}

Evolution predicts that homologous structures and functions of proteins exhibit a common descent proportional to the degree of their sequence similarity. Therefore, homology of primary amino acid sequences often serves as a lead to the function and the mechanism of proteins. However, a principal difficulty with MFS proteins in this respect is their low sequence homology. As mentioned above, it is likely that the detected $12-18 \%$ sequence homology results from overlapping hydrophobic residues that are not directly involved in the mechanism in most cases. Moreover, the inherent symmetry of the proteins with regard to the helix kinks and bends provides further nonspeciffic overlap [39]. Moreover, regions of functional similarity (e.g., substrateand/or $\mathrm{H}^{+}$-binding sites) do not align in less homologous MFS transporters; the functional counterparts are often located in distant parts of the protein (Figures 9(a) and 9(b)) $[45,141-143]$.

5.1. Helix Triplets. A clear picture of about a dozen MFS structures has unveiled X-ray crystallographically in only the past decade (Table 1), and recent studies suggest a key role for structural symmetry [144]. A recurring theme in transporters is an internal pseudosymmetry of (membrane spanning) helix sets $[50,145,146]$. Although structural and sequence symmetry was observed earlier $[15,51,89]$, the first functional implication was suggested by Forrest et al. [145]. Swapping the conformation of the two structurally similar repeats (TMs 1-5 and TMs 6-10), in the essentially outward-open conformation of LeuT, results in a hypothetical inward-open conformation. A similar approach has been used to generate a structural model of the outward-open LacY conformation [50]. Within the two six-helix domains related by a symmetry axis perpendicular to the membrane plane, two inverted-topology repeats are identified consisting of three-helix motifs. By swapping the conformations of inverted-topology repeats, an outward-open structure model is generated. Furthermore, the model agrees with DEER distance changes, accessibility to thiol reagents, and Cys cross-linking data [50]. The results are consistent with the hypothesis that MFS transporters may have arisen by intragenic multiplication of the triple-helix motif to form two pseudosymmetrical six-helix bundles $[49,51]$, the most common topological feature of MFS transporters.

5.2. Comparative Sequence-Function Analysis. One fundamentally important problem for understanding the mechanism of transport with active or passive transporters is the identification and physical localization of residues involved in substrate and ion binding. The broad sequence diversity of the MFS transporters presents a difficult problem in this respect. However, with a growing number of crystallographic structures, we can begin to identify functionally related residues, which are not aligned in the primary structures. The crystallographic model of FucP was obtained with the central cavity open to the periplasm [22] (Figure 9(a)), an outward-open conformation opposite to that of LacY [15-18]. Mutagenesis of Asp46 deep in the central cavity of FucP 


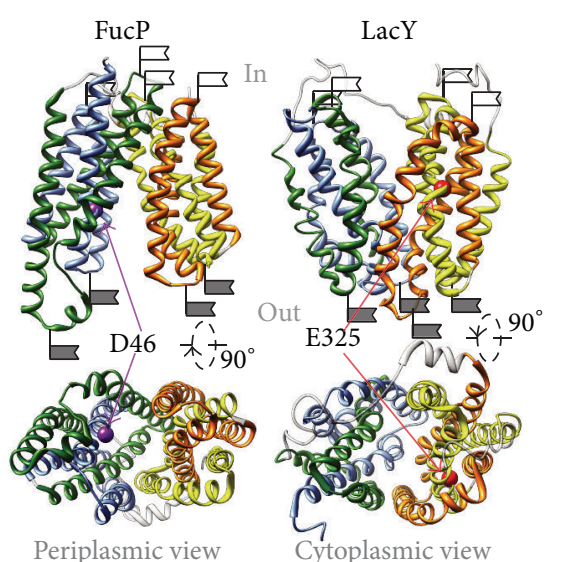

(a)

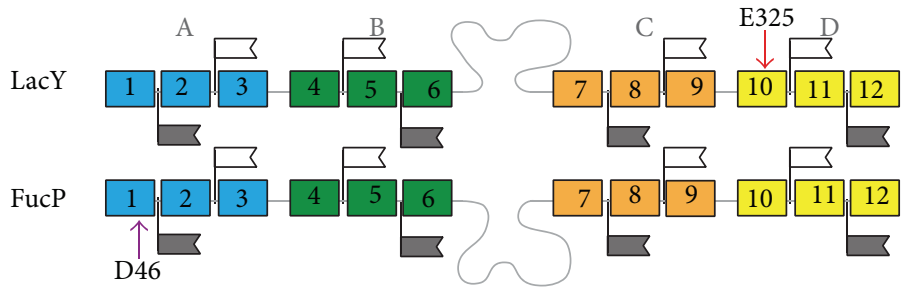

(b)

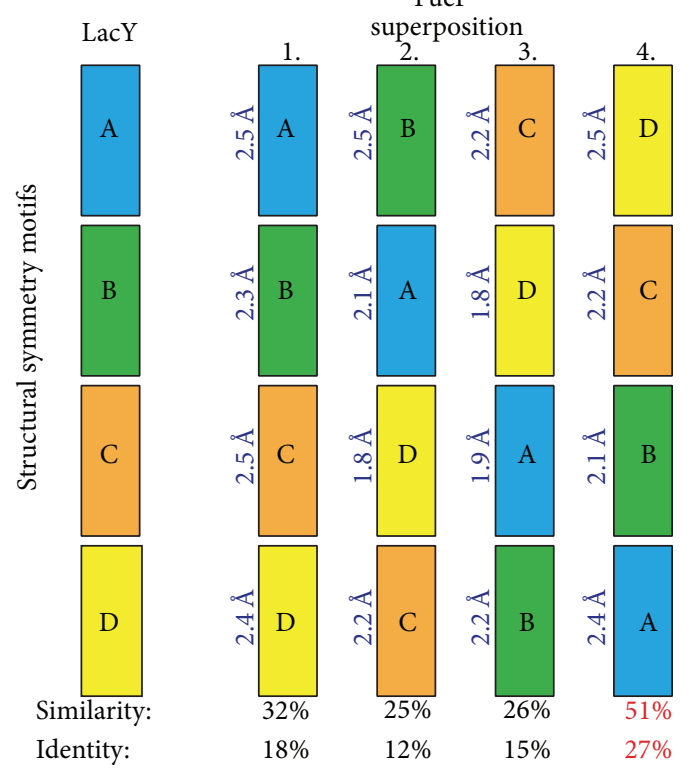

(c)

FIGURE 9: The evolutionary mix-and-match approach for the MFS. (a) Structure overview of FucP (PDBid 3O7Q) and LacY (PDBid 2V8N). Helices are color-coded according to the symmetry motifs: helices I-III: blue; helices IV-VI: green; helices VII-IX: orange; and helices X-XII: yellow. White and gray flags indicate cytoplasmic and periplasmic loops within the symmetry motifs, respectively. The positions of Asp46 (violet sphere in FucP) and Glu325 (red sphere in LacY) are indicated. (b) Schematic representation of the helix triplets in consecutive order in the sequence. Boxes represent the helices and are colored the same as in (a). The flags indicate the loops within symmetry motifs according to A and B. The positions of Asp46 and Glu325 are indicated by violet and red arrows, respectively. (c) Alternative aliments of FucP structure symmetry motifs on LacY structure symmetry motifs. Respective rmsds of the $\mathrm{C} \alpha$ atoms are indicated in blue for each superposition. The sequence similarity/identity for the combination of segment superimpositions for the 92 transport activity relevant positions (side-chain similarity matrix: AVLI, FYW, CM, ST, KRH, DENQ, and PG) is shown at the bottom. The best sequence alignment (superimposition 4) is highlighted in red.

completely abolishes L-fucose $/ \mathrm{H}^{+}$symport [22], but the mutants exhibit counterflow activity that is at least as good as WT [22], and they bind L-fucose with WT affinity [141]. The observations indicate that, like Glu325 mutants in LacY, Asp46 mutants in FucP are specifically blocked in reactions that do involve $\mathrm{H}^{+}$translocation [101]. Despite the related phenotypes, the location of the two carboxyl side chains in the respective proteins differs in location. Glu325 is located in the C-terminal six-helix bundle on helix X, while Asp46 is in the
N-terminal six-helix bundle on helix I (Figures 9(a) and 9(b)) [45]. A possible scenario leading to this discrepancy is that the MFS transporters are suggested to have arisen by intragenic multiplication and fusion of the structural motifs $[50,51]$. This concept is supported by the differing location of sidechains with similar phenotypes and it elicits the feasibility of alternative arrangements of the triple-helix motifs [45]. A comparative structure/sequence analysis permuting the consecutive order of the triple-helix motifs of LacY and 
FucP demonstrates that the strong symmetry within each transporter results in similar superimposition qualities with root mean square deviations (rmsd) ranging from $\sim 1.8 \AA$ to $\sim 2.6 \AA$. The respective sequence alignments of the triplehelix motifs are all in the same range of $\sim 25 \%$ similarity and do not provide any preference for a functional parity (Figure 9(c)) [45]. This is likely a result of the transporters disposition as $\alpha$-helical transmembrane protein; it consists of about $65 \%$ of hydrophobic residues, and a sequence alignment is biased by nonspecific hydrophobic overlap. However, if the conservation of residues in FucP is analyzed with respect to mutations in LacY that cause greater than $50 \%$ inhibition of the lactose transport rate [95], only one alignment combination exhibits a remarkable conservation of these functionally significant residues (Figure 9(c), 51\% similarity and $27 \%$ identity) [45]. In contrast to the conventional, linear sequence alignment, much stronger alignments between the sugar- and $\mathrm{H}^{+}$-binding sites in FucP and LacY are observed with the permuted order of the triplets of FucP (Figure 9(c): D-C-B-A). Although less than ten residues are absolutely irreplaceable, Cys substitution of 82 additional residues has a significant effect on activity, inhibiting the steady-state level of accumulation by $50-$ $80 \%$ in LacY. The alignment was tested experimentally for conservation by comparing the 92 LacY mutants that impair function with 34 analogous functional mutations in FucP [45]. An excellent correlation was detected for corresponding positions in the two proteins impairing the steady-state level of accumulation, and, interestingly, mutations at positions that do not have a significant influence on the substrate accumulation correlate as well. It appears that modern MFS symporters evolved from primordial noncovalently fused helix triplets, as functional mutations were being introduced and the functional segments assembled in a varying order. The idea suggests a simple, parsimonious chain of events that may have led to the enormous sequence diversity within the MFS. An alternative although less dynamic scenario is that a multispanning protein evolved first, possibly for stability reasons, and functional mutations were introduced later $[147,148]$. However, this scenario remains without any experimental support. In any case, using the notion of a "mix-and-match" evolution involving the triple-helix motifs in MFS, a more useful annotation of sequence motifs than a conventional, linear sequence alignment may be appropriate $[45,142,143]$.

5.3. A Common Mechanistic Pattern. The approach successfully used to compare FucP to LacY, in which the triplehelix motifs are aligned in combinatorial fashion, detects homologous residues in MFS members with known atomic structures that might be functionally related. Importantly, reported substrate and $\mathrm{H}^{+}$-binding sites in symporters that transport substrates, ranging from simple ions like phosphate to more complex peptides or disaccharides, are in similar locations and mutations display similar phenotypes $[45,142$, 143]. These observations strongly suggest that a common mechanistic pattern may be used for catalysis of symport by transporters in the MFS. A recent study comparing the structures and mutants of the bacterial sugar symporters, $\mathrm{XylE}$
(Xylose $/ \mathrm{H}^{+}$symporter from E. coli), and GlcP (glucose $/ \mathrm{H}^{+}$ symporter from $S$. epidermidis) to theoretical structures of the human glucose uniporters, the GLUTs, reveals that the general concepts derived from the bacterial transporters (Figure 8) (reviewed in [55]) are also relevant in the human GLUT uniporters [142]. The recent structural resolution of the human glucose transporter GLUT1 [36] verified the GLUT1 theoretical model (rmsd of the X-ray structure and the theoretical model: N-terminal domains: $1.105 \AA$ over $157 \mathrm{C} \alpha$ atoms, C-terminal domains: $1.037 \AA$ over $133 \mathrm{C} \alpha$ atoms, and ICH domain in the middle loop: $0.959 \AA$ over $28 \mathrm{C} \alpha$ atoms). This indicates that the functional side-chain correlations in GLUT1 concluded from positional homology of corresponding residues in $\mathrm{XylE}, \mathrm{GlcP}_{\mathrm{Se}}$, FucP, and LacY [142] are likely correct. XylE, FucP, and $\mathrm{GlcP}_{\text {Se }}$ have carboxyl groups corresponding to Glu325 in LacY that display a similar phenotype when neutralized by mutagenesis. The corresponding positions in GLUT1 (Asn29), which catalyzes facilitated diffusion, cannot be protonated, but many of the functionally important residues are conserved at homologous positions $[45,142,143]$. Therefore, it appears likely that the uniporter undergoes similar conformational transitions as symporters.

In summary, it is concluded that MFS transporters, including the GLUT uniporters, operate in a fashion analogous to enzymes with the exception that the intermediate is a conformer of the protein rather than a transition-state intermediate of the substrate. By this means, an intermediate occluded conformation is induced by sugar binding, and binding leads to lowering of the activation energy barriers for the transition between inwardand outward-open conformers.

\section{Conflict of Interests}

The author declares that there is no conflict of interests regarding the publication of this paper.

\section{Acknowledgments}

M. Gregor Madej is grateful to H. Ronald Kaback (UC Los Angeles) for editing the paper and for helpful comments on the paper. This work was supported by National Institutes of Health Grants DK51131, DK069463, and GM073210; National Science Foundation Grant MCB-1129551 (to H. Ronald Kaback ).

\section{References}

[1] G. N. Cohen and H. V. Rickenberg, "Etude directe de la fixation d'un inducteur de la b-galactosidase par les cellules d'Escherichia coli," Comptes Rendus Hebdomadaires des Séances de l'Académie des Sciences, vol. 240, pp. 466-468, 1955.

[2] B. Müller-Hill, The Lac Operon: A Short History of a Genetic Paradigm, Walter de Gruyter, Berlin, Germany, 1996.

[3] D. E. Buechel, B. Gronenborn, and B. Mueller-Hill, "Sequence of the lactose permease gene," Nature, vol. 283, no. 5747, pp. 541$545,1980$. 
[4] R. M. Teather, B. Müller-Hill, U. Abrutsch, G. Aichele, and P. Overath, "Amplification of the lactose carrier protein in Escherichia coli using a plasmid vector," MGG Molecular \& General Genetics, vol. 159, no. 3, pp. 239-248, 1978.

[5] G. J. Kaczorowski, G. LeBlanc, and H. R. Kaback, "Specific labeling of the lac carrier protein in membrane vesicles of Escherichia coli by a photoaffinity reagent," Proceedings of the National Academy of Sciences of the United States of America, vol. 77, no. 11 I, pp. 6319-6323, 1980.

[6] D. L. Foster, M. L. Garcia, M. J. Newman, L. Patel, and H. R. Kaback, "Lactose-proton symport by purified lac carrier protein," Biochemistry, vol. 21, no. 22, pp. 5634-5638, 1982.

[7] M. J. Newman, D. L. Foster, T. H. Wilson, and H. R. Kaback, "Purification and reconstitution of functional lactose carrier from Escherichia coli," The Journal of Biological Chemistry, vol. 256, no. 22, pp. 11804-11808, 1981.

[8] M. J. Newman and T. H. Wilson, "Solubilization and reconstitution of the lactose transport system from Escherichia coli," The Journal of Biological Chemistry, vol. 255, no. 22, pp. 10583-10586, 1980.

[9] P. Viitanen, M. L. Garcia, and H. R. Kaback, "Purified reconstituted lac carrier protein from Escherichia coli is fully functional," Proceedings of the National Academy of Sciences of the United States of America, vol. 81, no. 6, pp. 1629-1633, 1984.

[10] K. Matsushita, L. Patel, R. B. Gennis, and H. R. Kaback, "Reconstitution of active transport in proteoliposomes containing cytochrome o oxidase and lac carrier protein purified from Escherichia coli," Proceedings of the National Academy of Sciences of the United States of America, vol. 80, no. 16, pp. 4889-4893, 1983.

[11] P. Mitchell, "Molecule, group and electron transport through natural membranes," Biochemical Society Symposia, vol. 22, pp. 142-168, 1963.

[12] P. Mitchell, "Translocations through natural membranes," Advances in Enzymology and Related Areas of Molecular Biology, vol. 29, pp. 33-87, 1967.

[13] P. Mitchell, Chemiosmotic Coupling and Energy Transduction, Glynn Research, Bodmin, UK, 1968.

[14] H. R. Kaback, "Bacterial membranes," in Methods in Enzymol, N. P. Kaplan, W. B. Jakoby, and N. P. Colowick, Eds., vol. 22, pp. 99-120, Elsevier, New York, NY, USA, 1971.

[15] J. Abramson, I. Smirnova, V. Kasho, G. Verner, H. R. Kaback, and S. Iwata, "Structure and mechanism of the lactose permease of Escherichia coli," Science, vol. 301, no. 5633, pp. 610-615, 2003.

[16] V. Chaptal, S. Kwon, M. R. Sawaya, L. Guan, H. R. Kaback, and J. Abramson, "Crystal structure of lactose permease in complex with an affinity inactivator yields unique insight into sugar recognition," Proceedings of the National Academy of Sciences of the United States of America, vol. 108, no. 23, pp. 9361-9366, 2011.

[17] L. Guan, O. Mirza, G. Verner, S. Iwata, and H. R. Kaback, "Structural determination of wild-type lactose permease," Proceedings of the National Academy of Sciences of the United States of America, vol. 104, no. 39, pp. 15294-15298, 2007.

[18] O. Mirza, L. Guan, G. Verner, S. Iwata, and H. R. Kaback, "Structural evidence for induced fit and a mechanism for sugar $/ \mathrm{H}^{+}$symport in LacY," EMBO Journal, vol. 25, no. 6, pp. 1177-1183, 2006.

[19] H. Kumar, V. Kasho, I. Smirnova, J. S. Finer-Moore, H. R. Kaback, and R. M. Stroud, "Structure of sugar-bound LacY," Proceedings of the National Academy of Sciences of the United States of America, vol. 111, no. 5, pp. 1784-1788, 2014.
[20] Y. Huang, M. J. Lemieux, J. Song, M. Auer, and D.-N. Wang, "Structure and mechanism of the glycerol-3-phosphate transporter from Escherichia coli," Science, vol. 301, no. 5633, pp. 616620, 2003.

[21] Y. Yin, X. He, P. Szewczyk, T. Nguyen, and G. Chang, "Structure of the multidrug transporter EmrD from Escherichia coli," Science, vol. 312, no. 5774, pp. 741-744, 2006.

[22] S. Dang, L. Sun, Y. Huang et al., "Structure of a fucose transporter in an outward-open conformation," Nature, vol. 467, no. 7316, pp. 734-738, 2010.

[23] N. Solcan, J. Kwok, P. W. Fowler et al., "Alternating access mechanism in the POT family of oligopeptide transporters," The EMBO Journal, vol. 31, no. 16, pp. 3411-3421, 2012.

[24] S. Newstead, D. Drew, A. D. Cameron et al., "Crystal structure of a prokaryotic homologue of the mammalian oligopeptideproton symporters, PepT1 and PepT2," The EMBO Journal, vol. 30, no. 2, pp. 417-426, 2011.

[25] S. Doki, N. Solcan, M. Iwaki et al., "Structural basis for dynamic mechanism of proton-coupled symport by the peptide transporter POT,' Proceedings of the National Academy of Sciences of the United States of America, vol. 110, no. 28, pp. 11343-11348, 2013.

[26] F. Guettou, E. M. Quistgaard, L. Trésaugues et al., "Structural insights into substrate recognition in proton-dependent oligopeptide transporters," EMBO Reports, vol. 14, no. 9, pp. 804-810, 2013.

[27] L. Sun, X. Zeng, C. Yan et al., "Crystal structure of a bacterial homologue of glucose transporters GLUT1-4," Nature, vol. 490, no. 7420, pp. 361-366, 2012.

[28] E. M. Quistgaard, C. Löw, P. Moberg, L. Trésaugues, and P. Nordlund, "Structural basis for substrate transport in the GLUT-homology family of monosaccharide transporters," Nature Structural and Molecular Biology, vol. 20, no. 6, pp. 766768, 2013.

[29] B. P. Pedersen, H. Kumar, A. B. Waight et al., "Crystal structure of a eukaryotic phosphate transporter," Nature, vol. 496, no. 7446, pp. 533-536, 2013.

[30] C. V. Iancu, J. Zamoon, S. B. Woo, A. Aleshin, and J. Y. Choe, "Crystal structure of a glucose/H+ symporter and its mechanism of action," Proceedings of the National Academy of Sciences of the United States of America, vol. 110, no. 44, pp. 17862-17867, 2013.

[31] H. Yan, W. Huang, C. Yan et al., "Structure and mechanism of a nitrate transporter," Cell Reports, vol. 3, no. 3, pp. 716-723, 2013.

[32] H. Zheng, G. Wisedchaisri, and T. Gonen, "Crystal structure of a nitrate/nitrite exchanger," Nature, vol. 497, no. 7451, pp. 647651, 2013.

[33] A. S. Ethayathulla, M. S. Yousef, A. Amin, G. Leblanc, H. R. Kaback, and L. Guan, "Structure-based mechanism for $\mathrm{Na}(+) /$ melibiose symport by MelB," Nature communications, vol. 5, article 3009, 2014.

[34] J. L. Parker and S. Newstead, "Molecular basis of nitrate uptake by the plant nitrate transporter NRT1.1," Nature, vol. 507, no. 7490, pp. 68-72, 2014.

[35] J. Sun, "Crystal structure of the plant dual-affinity nitrate transporter NRT1.1," Nature, vol. 507, no. 7490, pp. 73-77, 2014.

[36] D. Deng, C. Xu, P. Sun et al., "Crystal structure of the human glucose transporter GLUT1," Nature, vol. 510, no. 7503, pp. 121125, 2014.

[37] P. Viitanen, M. L. Garcia, D. L. Foster, G. J. Kaczorowski, and H. R. Kaback, "Mechanism of lactose translocation in 
proteoliposomes reconstituted with lac carrier protein purified from Escherichia coli. 2. Deuterium solvent isotope effects," Biochemistry, vol. 22, no. 10, pp. 2531-2536, 1983.

[38] Z. Liu, M. G. Madej, and H. R. Kaback, "Helix Dynamics in LacY: helices II and IV," Journal of Molecular Biology, vol. 396, no. 3, pp. 617-626, 2010.

[39] M. G. Madej, S. N. Soro, and H. R. Kaback, "Apo-intermediate in the transport cycle of lactose permease (LacY)," Proceedings of the National Academy of Sciences of the United States of America, vol. 109, no. 44, pp. E2970-E2978, 2012.

[40] M. H. Saier Jr., "Families of transmembrane sugar transport proteins," Molecular Microbiology, vol. 35, no. 4, pp. 699-710, 2000.

[41] M. H. Saier Jr., J. T. Beatty, A. Goffeau et al., "The major facilitator superfamily," Journal of Molecular Microbiology and Biotechnology, vol. 1, no. 2, pp. 257-279, 1999.

[42] V. S. Reddy, M. A. Shlykov, R. Castillo, E. I. Sun, and M. H. Saier Jr., "The major facilitator superfamily (MFS) revisited," FEBS Journal, vol. 279, no. 11, pp. 2022-2035, 2012.

[43] V. N. Kasho, I. N. Smirnova, and H. R. Kaback, "Sequence alignment and homology threading reveals prokaryotic and eukaryotic proteins similar to lactose permease," Journal of Molecular Biology, vol. 358, no. 4, pp. 1060-1070, 2006.

[44] J. Sugihara, I. Smirnova, V. Kasho, and H. R. Kaback, "Sugar recognition by CscB and LacY," Biochemistry, vol. 50, no. 51, pp. 11009-11014, 2011.

[45] M. G. Madej, S. Dang, N. Yan, and H. R. Kaback, "Evolutionary mix-and-match with MFS transporters," Proceedings of the National Academy of Sciences of the United States of America, vol. 110, no. 15, pp. 5870-5874, 2013.

[46] D. Yernool, O. Boudker, Y. Jin, and E. Gouaux, "Structure of a glutamate transporter homologue from Pyrococcus horikoshii," Nature, vol. 431, no. 7010, pp. 811-818, 2004.

[47] C. Hunte, E. Screpanti, M. Venturi, A. Rimon, E. Padan, and H. Michel, "Structure of a Na+/H+ antiporter and insights into mechanism of action and regulation by pH," Nature, vol. 435, no. 7046, pp. 1197-1202, 2005.

[48] Y. Shi, "Common folds and transport mechanisms of secondary active transporters," Annual Review of Biophysics, vol. 42, no. 1, pp. 51-72, 2013.

[49] S. S. Pao, I. T. Paulsen, and M. H. Saier Jr., "Major facilitator superfamily," Microbiology and Molecular Biology Reviews, vol. 62, no. 1, pp. 1-34, 1998.

[50] S. Radestock and L. R. Forrest, "The alternating-access mechanism of MFS transporters arises from inverted-topology repeats," Journal of Molecular Biology, vol. 407, no. 5, pp. 698715, 2011.

[51] R. N. Hvorup and M. H. Saier, "Sequence similarity between the channel-forming domains of voltage-gated ion channel proteins and the C-terminal domains of secondary carriers of the major facilitator superfamily," Microbiology, vol. 148, part 12, pp. 37603762, 2002.

[52] L. Guan and H. R. Kaback, "Lessons from lactose permease," Annual Review of Biophysics and Biomolecular Structure, vol. 35, pp. 67-91, 2006.

[53] H. R. Kaback, S. Frillingos, H. Jung et al., "The lactose permease meets Frankenstein," The Journal of Experimental Biology, vol. 196, pp. 183-195, 1994.

[54] H. R. Kaback, M. Sahin-Tóth, and A. B. Weinglass, "The kamikaze approach to membrane transport," Nature Reviews Molecular Cell Biology, vol. 2, no. 8, pp. 610-620, 2001.
[55] M. G. Madej and H. R. Kaback, "The life and times of lac permease: crystals Ain't enough, but they certainly do help," in Membrane Transporter Function: To Structure and Beyond, C. Ziegler and R. Kraemer, Eds., vol. 17 of Springer Series in Biophysics: Transporters, pp. 121-158, 2014.

[56] I. Smirnova, V. Kasho, and H. R. Kaback, "Lactose permease and the alternating access mechanism," Biochemistry, vol. 50, no. 45, pp. 9684-9693, 2011.

[57] J. Calamia and C. Manoil, "lac permease of Escherichia coli: topology and sequence elements promoting membrane insertion," Proceedings of the National Academy of Sciences of the United States of America, vol. 87, no. 13, pp. 4937-4941, 1990.

[58] G. G. Privé and H. R. Kaback, "Engineering the lac permease for purification and crystallization," Journal of Bioenergetics and Biomembranes, vol. 28, no. 1, pp. 29-34, 1996.

[59] A. B. Weinglass and H. R. Kaback, "The central cytoplasmic loop of the major facilitator superfamily of transport proteins governs efficient membrane insertion," Proceedings of the National Academy of Sciences of the United States of America, vol. 97, no. 16, pp. 8938-8943, 2000.

[60] C. D. Wolin and H. R. Kaback, "Estimating loop-helix interfaces in a polytopic membrane protein by deletion analysis," Biochemistry, vol. 38, no. 26, pp. 8590-8597, 1999.

[61] L. Guan and H. R. Kaback, "Site-directed alkylation of cysteine to test solvent accessibility of membrane proteins," Nature Protocols, vol. 2, no. 8, pp. 2012-2017, 2007.

[62] D. L. Foster, M. Boublik, and H. R. Kaback, "Structure of the lac carrier protein of Escherichia coli," Journal of Biological Chemistry, vol. 258, no. 1, pp. 31-34, 1983.

[63] H. Vogel, J. K. Wright, and F. Jähnig, "The structure of the lactose permease derived from Raman spectroscopy and prediction methods," EMBO Journal A, vol. 4, no. 13, pp. 36253631, 1985.

[64] J. le Coutre, L. R. Narasimhan, C. K. N. Patel, and H. R. Kaback, "The lipid bilayer determines helical tilt angle and function in lactose permease of Escherichia coli," Proceedings of the National Academy of Sciences of the United States of America, vol. 94, no. 19, pp. 10167-10171, 1997.

[65] S. C. King, C. L. Hansen, and T. H. Wilson, "The interaction between aspartic acid 237 and lysine 358 in the lactose carrier of Escherichia coli," Biochimica et Biophysica Acta: Biomembranes, vol. 1062, no. 2, pp. 177-186, 1991.

[66] S. C. King and S. Li, "Suppressor scanning at positions 177 and 236 in the Escherichia coli lactose/H+ cotransporter and stereotypical effects of acidic substituents that suggest a favored orientation of transmembrane segments relative to the lipid bilayer," Journal of Bacteriology, vol. 180, no. 10, pp. 2756-2758, 1998.

[67] J. Wu and H. R. Kaback, "A general method for determining helix packing in membrane proteins in situ: hHelices I and II are close to helix VII in the lactose permease of Escherichia coli," Proceedings of the National Academy of Sciences of the United States of America, vol. 93, no. 25, pp. 14498-14502, 1996.

[68] Y. Zhou, L. Guan, J. A. Freites, and H. R. Kaback, "Opening and closing of the periplasmic gate in lactose permease," Proceedings of the National Academy of Sciences of the United States of America, vol. 105, no. 10, pp. 3774-3778, 2008.

[69] K. Jung, H. Jung, J. Wu, G. G. Privé, and H. R. Kaback, "Use of site-directed fluorescence labeling to study proximity relationships in the lactose permease of Escherichia coli," Biochemistry, vol. 32, no. 46, pp. 12273-12278, 1993. 
[70] K. Jung, J. Voss, M. He, W. L. Hubbell, and H. R. Kaback, "Engineering a metal binding site within a polytopic membrane protein, the lactose permease of Escherichia coli," Biochemistry, vol. 34, no. 19, pp. 6272-6277, 1995.

[71] M. Zhao, K.-C. Zen, J. Hernandez-Borrell, C. Altenbach, W. L. Hubbell, and H. R. Kaback, "Nitroxide scanning electron paramagnetic resonance of helices IV and V and the intervening loop in the lactose permease of Escherichia coli," Biochemistry, vol. 38, no. 48, pp. 15970-15977, 1999.

[72] I. Smirnova, V. Kasho, J.-Y. Choe, C. Altenbach, W. L. Hubbell, and H. R. Kaback, "Sugar binding induces an outward facing conformation of LacY," Proceedings of the National Academy of Sciences of the United States of America, vol. 104, no. 42, pp. 16504-16509, 2007.

[73] J. Sun, J. Li, N. Carrasco, and H. R. Kaback, "The last two cytoplasmic loops in the lactose permease of Escherichia coli comprise a discontinuous epitope for a monoclonal antibody," Biochemistry, vol. 36, no. 1, pp. 274-280, 1997.

[74] J. Sun, J. Wu, N. Carrasco, and H. R. Kaback, "Identification of the epitope for monoclonal antibody $4 \mathrm{~B} 1$ which uncouples lactose and proton translocation in the lactose permease of Escherichia coli," Biochemistry, vol. 35, no. 3, pp. 990-998, 1996.

[75] G. J. Kaczorowski, "Mechanism of lactose translocation in membrane vesicles from Escherichia coli. 1. Effect of $\mathrm{pH}$ on efflux, exchange, and counterflow," Biochemistry, vol. 18, no. 17, pp. 3691-3697, 1979.

[76] H. R. Kaback, I. Smirnova, V. Kasho, Y. Nie, and Y. Zhou, “The alternating access transport mechanism in LacY," The Journal of Membrane Biology, vol. 239, no. 1-2, pp. 85-93, 2011.

[77] I. Smirnova, V. Kasho, and H. R. Kaback, "Real-time conformational changes in LacY," Proceedings of the National Academy of Sciences of the United States of America, vol. 111, no. 23, pp. 8440-8445, 2014.

[78] P. Mitchell, "A general theory of membrane transport from studies of bacteria," Nature, vol. 180, no. 4577, pp. 134-136, 1957.

[79] M. Pannier, S. Veit, A. Godt, G. Jeschke, and H. W. Spiess, "Dead-time free measurement of dipole-dipole interactions between electron spins," Journal of Magnetic Resonance, vol. 142, no. 2, pp. 331-340, 2000.

[80] G. Jeschke, "Distance measurements in the nanometer range by pulse EPR," Chemphyschem, vol. 3, no. 11, pp. 927-932, 2002.

[81] C. J. Law, P. C. Maloney, and D.-N. Wang, "Ins and outs of major facilitator superfamily antiporters," Annual Review of Microbiology, vol. 62, pp. 289-305, 2008.

[82] P. J. F. Henderson and S. A. Baldwin, "This is about the in and the out," Nature Structural and Molecular Biology, vol. 20, no. 6, pp. 654-655, 2013.

[83] J. Le Coutre, H. R. Kaback, C. K. N. Patel, L. Heginbotham, and C. Miller, "Fourier transform infrared spectroscopy reveals a rigid $\alpha$-helical assembly for the tetrameric Streptomyces lividans K+ channel," Proceedings of the National Academy of Sciences of the United States of America, vol. 95, no. 11, pp. 61146117, 1998.

[84] J. S. Patzlaff, J. A. Moeller, B. A. Barry, and R. J. Brooker, "Fourier transform infrared analysis of purified lactose permease: a monodisperse lactose permease preparation is stably folded, $\alpha$-helical, and highly accessible to deuterium exchange," Biochemistry, vol. 37, no. 44, pp. 15363-15375, 1998.

[85] W. M. H. Sayeed and J. E. Baenziger, "Structural characterization of the osmosensor ProP," Biochimica et Biophysica Acta, vol. 1788, no. 5, pp. 1108-1115, 2009.
[86] Y. Zhou, M. G. Madej, L. Guan, Y. Nie, and H. R. Kaback, "An early event in the transport mechanism of LacY protein: interaction between helices V and I," The Journal of Biological Chemistry, vol. 286, no. 35, pp. 30415-30422, 2011.

[87] W. Zhang, L. Guan, and H. R. Kaback, "Helices VII and X in the lactose permease of Escherichia coli: proximity and ligandinduced distance changes," Journal of Molecular Biology, vol. 315, no. 1, pp. 53-62, 2002.

[88] L. S. Stelzl, P. W. Fowler, M. S. Sansom, and O. Beckstein, "Flexible gates generate occluded intermediates in the transport cycle of LacY," Journal of Molecular Biology, vol. 426, no. 3, pp. 735-751, 2014.

[89] T. Hirai, J. A. W. Heymann, D. Shi, R. Sarker, P. C. Maloney, and S. Subramaniam, "Three-dimensional structure of a bacterial oxalate transporter," Nature Structural Biology, vol. 9, no. 8, pp. 597-600, 2002.

[90] I. Smirnova, V. Kasho, J. Sugihara, and H. R. Kaback, “Trp replacements for tightly interacting Gly-Gly pairs in LacY stabilize an outward-facing conformation," Proceedings of the National Academy of Sciences of the United States of America, vol. 110, no. 22, pp. 8876-8881, 2013.

[91] C. F. Fox and E. P. Kennedy, "Specific labeling and partial purification of the $\mathrm{M}$ protein, a component of the betagalactoside transport system of Escherichia coli," Proceedings of the National Academy of Sciences of the United States of America, vol. 54, no. 3, pp. 891-899, 1965.

[92] B. Bieseler, H. Prinz, and K. Beyreuther, "Topological studies of lactose permease of Escherichia coli by protein sequence analysis," Annals of the New York Academy of Sciences, vol. 456, pp. 309-341, 1985.

[93] W. R. Trumble, P. V. Viitanen, H. K. Sarkar, M. S. Poonian, and H. R. Kaback, "Site-directed mutagenesis of cys148 in the lac carrier protein of Escherichia coli," Biochemical and Biophysical Research Communications, vol. 119, no. 3, pp. 860-867, 1984.

[94] H. Jung, K. Jung, and H. R. Kaback, "Cysteine 148 in the lactose permease of Escherichia coli is a component of a substrate binding site. 1. Site-directed mutagenesis studies," Biochemistry, vol. 33, no. 40, pp. 12160-12165, 1994.

[95] S. Frillingos, M. Sahin-Tóth, J. Wu, and H. R. Kaback, "Cysscanning mutagenesis: a novel approach to structure-function relationships in polytopic membrane proteins," The FASEB Journal, vol. 12, no. 13, pp. 1281-1299, 1998.

[96] L. Guan, Y. Hu, and H. R. Kaback, "Aromatic stacking in the sugar binding site of the lactose permease," Biochemistry, vol. 42, no. 6, pp. 1377-1382, 2003.

[97] J. L. Vázquez-Ibar, L. Guan, M. Svrakic, and H. R. Kaback, "Exploiting luminescence spectroscopy to elucidate the interaction between sugar and a tryptophan residue in the lactose permease of Escherichia coli," Proceedings of the National Academy of Sciences of the United States of America, vol. 100, no. 22, pp. 12706-12711, 2003.

[98] M. Sahin-Tóth, J. Le Coutre, D. Kharabi, G. Le Maire, J. C. Lee, and H. R. Kaback, "Characterization of Glu126 and Arg144, two residues that are indispensable for substrate binding in the lactose permease of Escherichia coli," Biochemistry, vol. 38, no. 2, pp. 813-819, 1999.

[99] S. Frillingos, A. Gonzalez, and H. R. Kaback, "Cysteinescanning mutagenesis of helix IV and the adjoining loops in the lactose permease of Escherichia coli: Glu126 and Arg144 are essential," Biochemistry, vol. 36, no. 47, pp. 14284-14290, 1997.

[100] P. Venkatesan and H. R. Kaback, "The substrate-binding site in the lactose permease of Escherichia coli," Proceedings of the 
National Academy of Sciences of the United States of America, vol. 95, no. 17, pp. 9802-9807, 1998.

[101] I. Smirnova, V. Kasho, J. Sugihara, J.-Y. Choe, and H. R. Kaback, "Residues in the $\mathrm{H}^{+}$translocation site define the $\mathrm{p} K_{a}$ for sugar binding to LacY," Biochemistry, vol. 48, no. 37, pp. 8852-8860, 2009.

[102] X. Jiang, M. K. Villafuerte, M. Andersson, S. H. White, and H. R. Kaback, "Galactoside-binding site in LacY," Biochemistry, vol. 53, no. 9, pp. 1536-1543, 2014.

[103] M. Sahin-Tóth, K. M. Akhoon, J. Runner, and H. R. Kaback, "Ligand recognition by the lactose permease of Escherichia coli: specificity and affinity are defined by distinct structural elements of galactopyranosides," Biochemistry, vol. 39, no. 17, pp. 5097-5103, 2000.

[104] M. Sahin-Tóth, M. C. Lawrence, T. Nishio, and H. R. Kaback, "The C-4 hydroxyl group of galactopyranosides is the major determinant for ligand recognition by the lactose permease of Escherichia coli," Biochemistry, vol. 40, no. 43, pp. 13015-13019, 2001.

[105] E. Padan, H. K. Sarkar, P. V. Viitanen, M. S. Poonian, and H. R. Kaback, "Site-specific mutagenesis of histidine residues in the lac permease of Escherichia coli," Proceedings of the National Academy of Sciences of the United States of America, vol. 82, no. 20, pp. 6765-6768, 1985.

[106] I. B. Püttner, H. K. Sarkar, M. S. Poonian, and H. R. Kaback, "Lac permease of Escherichia coli: histidine-205 and histidine322 play different roles in lactose/ $\mathrm{H}+$ symport," Biochemistry, vol. 25, no. 16, pp. 4483-4485, 1986.

[107] N. Carrasco, L. M. Antes, M. S. Poonian, and H. Ronald Kaback, "Lac permease of Escherichia coli: histidine-322 and glutamic acid-325 may be components of a charge-relay system," Biochemistry, vol. 25, no. 16, pp. 4486-4488, 1986.

[108] N. Carrasco, I. B. Püttner, L. M. Antes et al., "Characterization of site-directed mutants in the lac permease of Escherichia coli. 2. Glutamate-325 replacements," Biochemistry, vol. 28, no. 6, pp. 2533-2539, 1989.

[109] M. Sahin-Tóth and H. R. Kaback, "Arg-302 facilitates deprotonation of Glu-325 in the transport mechanism of the lactose permease from Escherichia coli," Proceedings of the National Academy of Sciences of the United States of America, vol. 98, no. 11, pp. 6068-6073, 2001.

[110] M. M. He, J. Voss, W. L. Hubbell, and H. Ronald Kaback, "Use of designed metal-binding sites to study helix proximity in the lactose permease of Escherichia coli. 2. Proximity of helix IX (Arg302) with helix X (His322 and Glu325)," Biochemistry, vol. 34, no. 48, pp. 15667-15670, 1995.

[111] L. Guan, M. Sahin-Tóth, and H. R. Kaback, "Changing the lactose permease of Escherichia coli into a galactose-specific symporter," Proceedings of the National Academy of Sciences of the United States of America, vol. 99, no. 10, pp. 6613-6618, 2002.

[112] T. G. Consler, O. Tsolas, and H. R. Kaback, "Role of proline residues in the structure and function of a membrane transport protein," Biochemistry, vol. 30, no. 5, pp. 1291-1298, 1991.

[113] K. Jung, H. Jung, P. Colacurcio, and H. R. Kaback, "Role of glycine residues in the structure and function of lactose permease, an Escherichia coli membrane transport protein," Biochemistry, vol. 34, no. 3, pp. 1030-1039, 1995.

[114] D. N. Langelaan, M. Wieczorek, C. Blouin, and J. K. Rainey, "Improved helix and kink characterization in membrane proteins allows evaluation of kink sequence predictors," Journal of Chemical Information and Modeling, vol. 50, no. 12, pp. 22132220, 2010.
[115] N. V. Ermolova, I. N. Smirnova, V. N. Kasho, and H. R. Kaback, "Interhelical packing modulates conformational flexibility in the lactose permease of Escherichia coli," Biochemistry, vol. 44, no. 21, pp. 7669-7677, 2005.

[116] T. Serdiuk, M. G. Madej, J. Sugihara et al., "Substrate-induced changes in the structural properties of LacY" Proceedings of the National Academy of Sciences of the United States of America, vol. 111, no. 16, pp. E1571-E1580, 2014.

[117] R. L. Dunten, M. Sahin-Tóth, and H. R. Kaback, "Role of the charge pair aspartic acid-237-lysine-358 in the lactose permease of Escherichia coli," Biochemistry, vol. 32, no. 12, pp. 3139-3145, 1993.

[118] M. Sahin-Tóth and H. R. Kaback, "Properties of interacting aspartic acid and lysine residues in the lactose permease of Escherichia coli," Biochemistry, vol. 32, no. 38, pp. 10027-10035, 1993.

[119] M. Sahin-Toth, R. L. Dunten, A. Gonzalez, and H. R. Kaback, "Functional interactions between putative intramembrane charged residues in the lactose permease of Escherichia coli," Proceedings of the National Academy of Sciences of the United States of America, vol. 89, no. 21, pp. 10547-10551, 1992.

[120] B. Persson, P. D. Roepe, L. Patel, J. Lee, and H. R. Kaback, "Sitedirected mutagenesis of lysine 319 in the lactose permease of Escherichia coli," Biochemistry, vol. 31, no. 37, pp. 8892-8897, 1992.

[121] I. Smirnova, V. Kasho, J. Sugihara, J. L. Vázquez-Ibar, and H. R. Kaback, "Role of protons in sugar binding to LacY," Proceedings of the National Academy of Sciences of the United States of America, vol. 109, no. 42, pp. 16835-16840, 2012.

[122] Y. Nie and H. R. Kaback, "Sugar binding induces the same global conformational change in purified LacY as in the native bacterial membrane," Proceedings of the National Academy of Sciences of the United States of America, vol. 107, no. 21, pp. 99039908, 2010.

[123] X. Jiang, A. J. Driessen, B. L. Feringa, and H. R. Kaback, “The periplasmic cavity of LacY mutant Cys154 $\rightarrow$ Gly: how open is open?" Biochemistry, vol. 52, no. 37, pp. 6568-6574, 2013.

[124] I. N. Smirnova, V. N. Kasho, and H. R. Kaback, "Direct sugar binding to LacY measured by resonance energy transfer," Biochemistry, vol. 45, no. 51, pp. 15279-15287, 2006.

[125] I. Smirnova, V. Kasho, J. Sugihara, and H. R. Kaback, “Opening the periplasmic cavity in lactose permease is the limiting step for sugar binding," Proceedings of the National Academy of Sciences of the United States of America, vol. 108, no. 37, pp. 15147-15151, 2011.

[126] M. Sahin-Tóth, A. Karlin, and H. R. Kaback, "Unraveling the mechanism of the lactose permease of Escherichia coli," Proceedings of the National Academy of Sciences of the United States of America, vol. 97, no. 20, pp. 10729-10732, 2000.

[127] I. N. Smirnova, V. Kasho, and H. R. Kaback, "Protonation and sugar binding to LacY," Proceedings of the National Academy of Sciences of the United States of America, vol. 105, no. 26, pp. 8896-8901, 2008.

[128] M. Soskine, Y. Adam, and S. Schuldiner, "Direct evidence for substrate-induced proton release in detergent-solubilized EmrE, a multidrug transporter," The Journal of Biological Chemistry, vol. 279, no. 11, pp. 9951-9955, 2004.

[129] D. E. Robertson, "Mechanism of lactose translocation in membrane vesicles from Escherichia coli. 2. Effect of imposed delta psi, delta $\mathrm{pH}$, and delta mu H+," Biochemistry, vol. 18, no. 17, pp. 3697-3704, 1979. 
[130] W. P. Jenks, Catalysis in Chemistry and Enzymology, McGrawHill, New York, NY, USA, 1969.

[131] R. L. Schowen, Isotope Effects on Enzymes-Catalyzed Reactions, University Park Press, Baltimore, Md, USA, 1977.

[132] N. Carrasco, P. Viitanen, D. Herzlinger, and H. R. Kaback, "Monoclonal antibodies against the lac carrier protein from Escherichia coli. I. Functional studies," Biochemistry, vol. 23, no. 16, pp. 3681-3687, 1984.

[133] J. J. Garcia-Celma, I. N. Smirnova, H. R. Kaback, and K. Fendler, "Electrophysiological characterization of LacY," Proceedings of the National Academy of Sciences of the United States of America, vol. 106, no. 18, pp. 7373-7378, 2009.

[134] J. J. Garcia-Celma, J. Ploch, I. Smirnova, H. R. Kaback, and K. Fendler, "Delineating electrogenic reactions during lactose $/ \mathrm{H}^{+}$ symport," Biochemistry, vol. 49, no. 29, pp. 6115-6121, 2010.

[135] O. Gaiko, A. Bazzone, K. Fendler, and H. R. Kaback, "Electrophysiological characterization of uncoupled mutants of LacY," Biochemistry, vol. 52, no. 46, pp. 8261-8266, 2013.

[136] Y. Zhou, X. Jiang, and H. R. Kaback, "Role of the irreplaceable residues in the LacY alternating access mechanism," Proceedings of the National Academy of Sciences of the United States of America, vol. 109, no. 31, pp. 12438-12442, 2012.

[137] M. Andersson, A. N. Bondar, J. A. Freites, D. J. Tobias, H. R. Kaback, and S. H. White, "Proton-coupled dynamics in lactose permease," Structure, vol. 20, no. 11, pp. 1893-1904, 2012.

[138] L. Guan and H. R. Kaback, "Binding affinity of lactose permease is not altered by the $\mathrm{H}+$ electrochemical gradient," Proceedings of the National Academy of Sciences of the United States of America, vol. 101, no. 33, pp. 12148-12152, 2004.

[139] D. E. Robertson, G. J. Kaczorowski, M.-L. Garcia, and H. R. Kaback, "Active transport in membrane vesicles from Escherichia coli: the electrochemical proton gradient alters the distribution of the lac carrier between two different kinetic states," Biochemistry, vol. 19, no. 25, pp. 5692-5702, 1980.

[140] Y. Nie, I. Smirnova, V. Kasho, and H. R. Kaback, "Energetics of ligand-induced conformational flexibility in the lactose permease of Escherichia coli," Journal of Biological Chemistry, vol. 281, no. 47, pp. 35779-35784, 2006.

[141] J. Sugihara, L. Sun, N. Yan, and H. R. Kaback, "Dynamics of the L-fucose/H+ symporter revealed by fluorescence spectroscopy," Proceedings of the National Academy of Sciences of the United States of America, vol. 109, no. 37, pp. 14847-14851, 2012.

[142] M. G. Madej, L. Sun, N. Yan, and H. R. Kaback, "Functional architecture of MFS D-glucose transporters," Proceedings of the National Academy of Sciences of the United States of America, vol. 111, no. 7, pp. E719-E727, 2014.

[143] M. G. Madej and H. R. Kaback, "Evolutionary mix-and-match with MFS transporters II," Proceedings of the National Academy of Sciences of the United States of America, vol. 110, no. 50, pp. E4831-E4838.

[144] L. R. Forrest, "Structural biology: (pseudo-)symmetrical transport," Science, vol. 339, no. 6118, pp. 399-401, 2013.

[145] L. R. Forrest, Y.-W. Zhang, M. T. Jacobs et al., "Mechanism for alternating access in neurotransmitter transporters," Proceedings of the National Academy of Sciences of the United States of America, vol. 105, no. 30, pp. 10338-10343, 2008.

[146] T. J. Crisman, S. Qu, B. I. Kanner, and L. R. Forrest, "Inwardfacing conformation of glutamate transporters as revealed by their inverted-topology structural repeats," Proceedings of the National Academy of Sciences of the United States of America, vol. 106, no. 49, pp. 20752-20757, 2009.
[147] A. Vastermark, B. Lunt, and M. Saier, "Major facilitator superfamily porters, LacY, FucP and XylE of Escherichia coli appear to have evolved positionally dissimilar catalytic residues without rearrangement of 3-TMS repeat units," Journal of Molecular Microbiology and Biotechnology, vol. 24, no. 2, pp. 82-90, 2014.

[148] A. Vastermark and M. H. Saier, "Major Facilitator Superfamily (MFS) evolved without 3-transmembrane segment unit rearran gements," Proceedings of the National Academy of Sciences of the United States of America, vol. 111, no. 13, pp. E1162-E1163, 2014. 

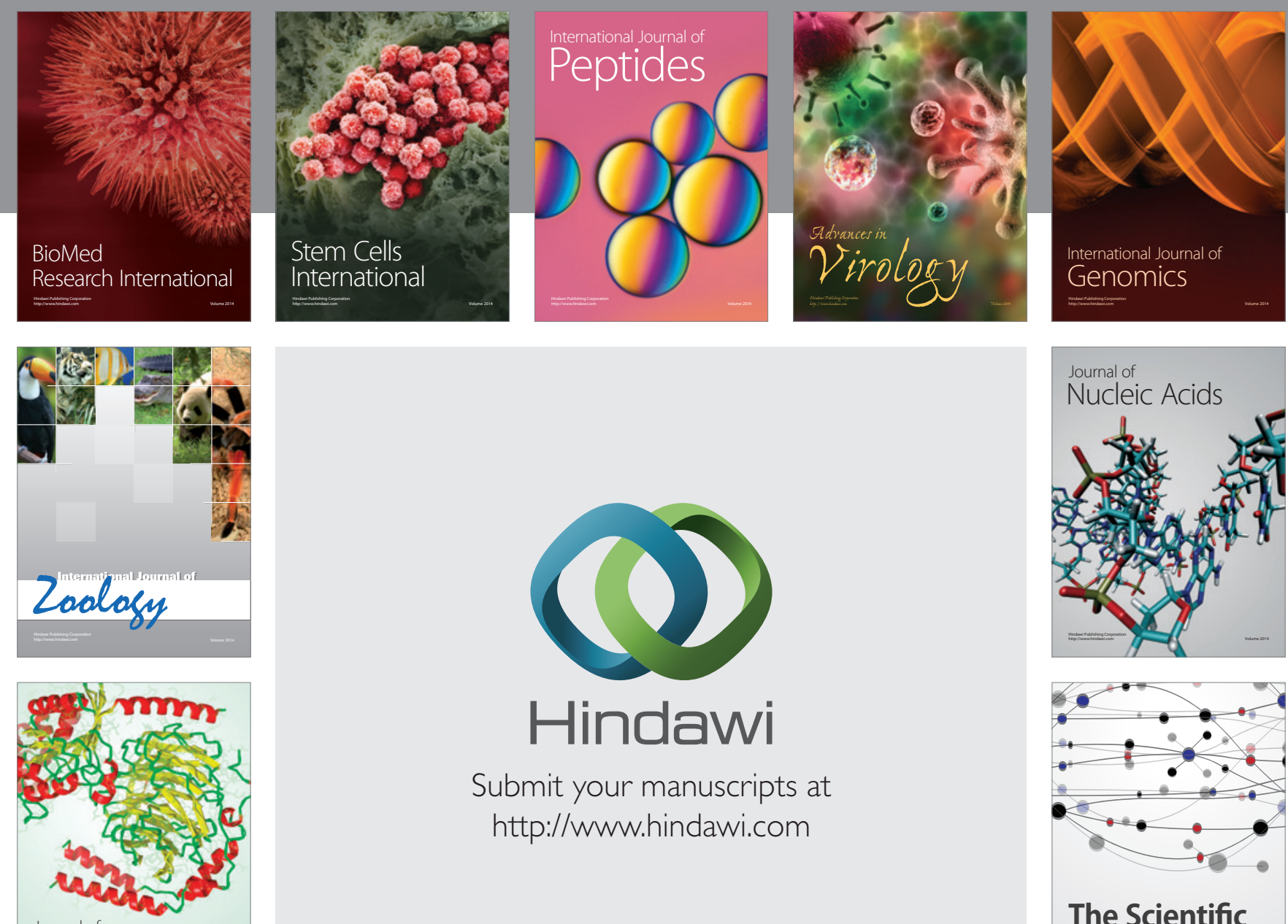

Submit your manuscripts at

http://www.hindawi.com

Journal of
Signal Transduction
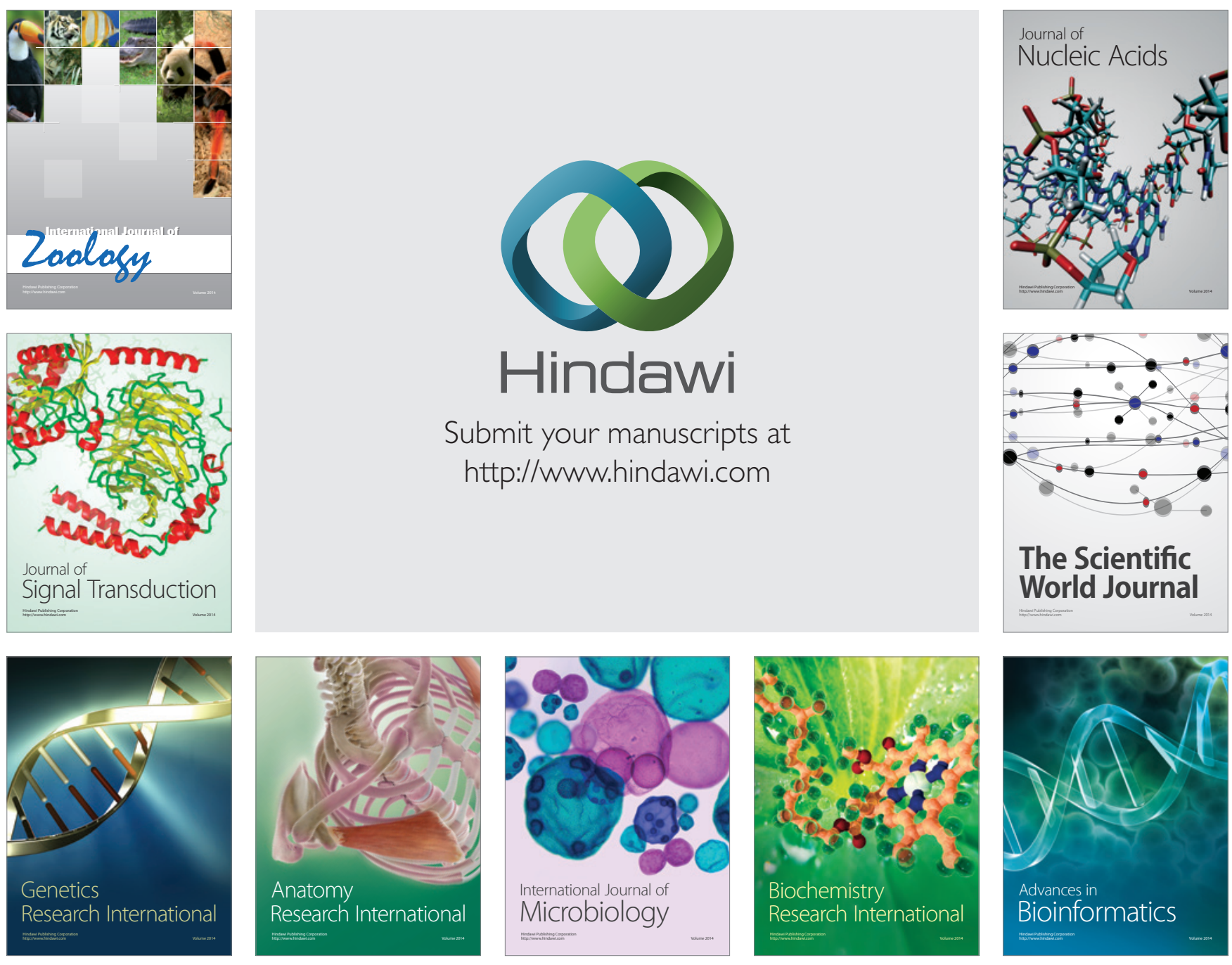

The Scientific World Journal
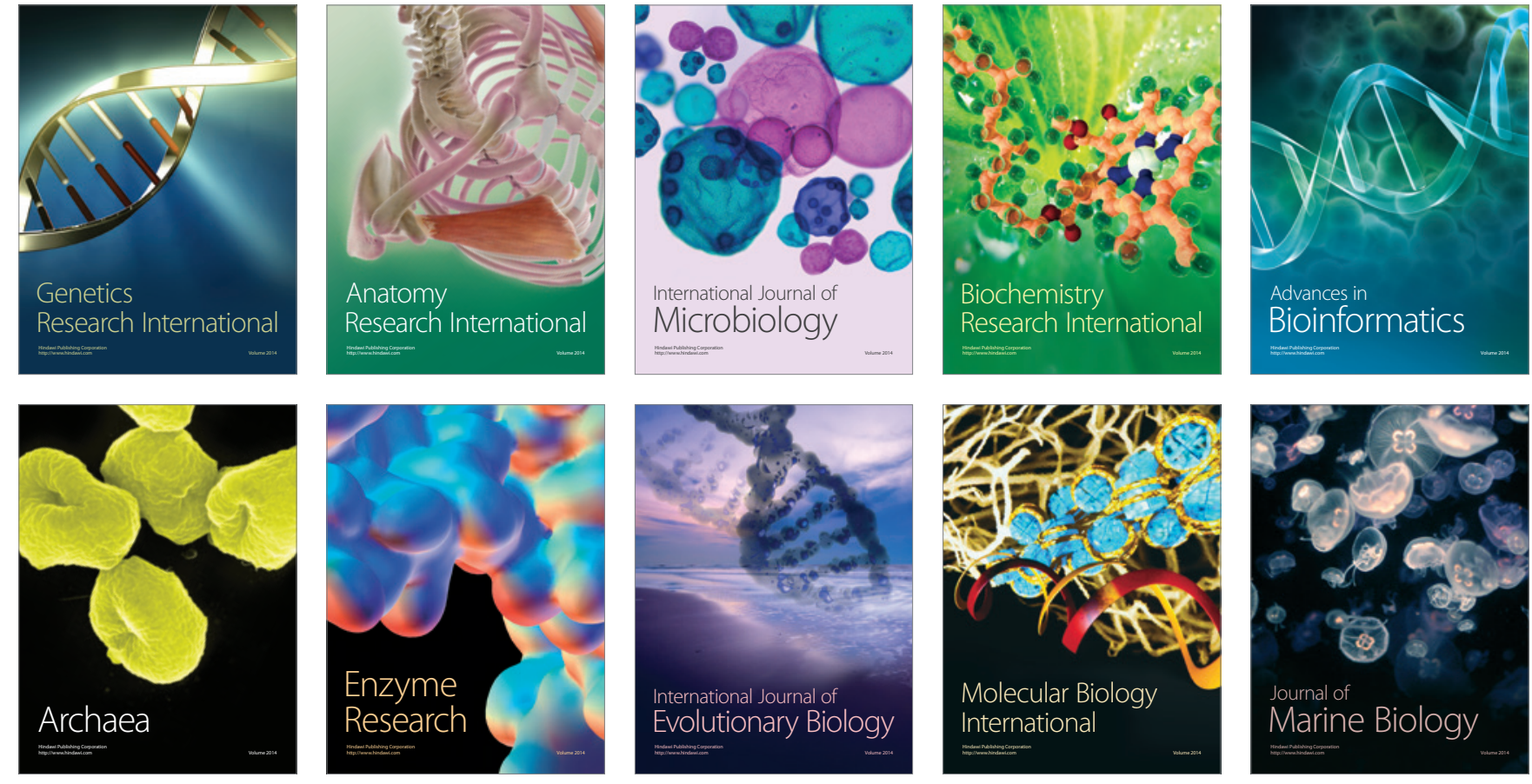